\title{
Cross-field flow and electric potential in a plasma slab
}

\author{
J. De Keyser, M. Echim, and M. Roth \\ Belgian Institute for Space Aeronomy (BIRA-IASB), Ringlaan 3, 1180 Brussels, Belgium \\ Correspondence to: J. De Keyser (johan.dekeyser@aeronomie.be)
}

Received: 13 May 2013 - Revised: 28 June 2013 - Accepted: 1 July 2013 - Published: 1 August 2013

\begin{abstract}
We consider cross-field plasma flow inside a fieldaligned plasma slab embedded in a uniform background in a 1-dimensional geometry. This situation may arise, for instance, when long-lasting reconnection pulses inject plasma into the inner magnetosphere. The present paper presents a detailed analysis of the structure of the interfaces that separate the slab from the background plasma on either side; a fully kinetic model is used to do so. Since the velocity shear across both interfaces has opposite signs, and given the typical gyroradius differences between injected and background ions and electrons, the structure of both interfaces can be very different. The behaviour of the slab and its interfaces depends critically on the flow of the plasma transverse to the magnetic field; in particular, it is shown that there are bounds to the flow speed that can be supported by the magnetised plasma. Further complicating the picture is the effect of the potential difference between the slab and its environment.
\end{abstract}

Keywords. Magnetospheric physics (plasma sheet) - Space plasma physics (discontinuities; kinetic and MHD theory)

\section{Introduction}

This paper deals with a 1-dimensional idealised plasma and magnetic field configuration that can serve as a model for a number of situations that occur in the magnetosphere. We consider a plasma slab with a certain thickness that is embedded in a background plasma pervaded by a strong background magnetic field (low plasma $\beta$ ). The plasma flows in the slab in the direction perpendicular to the field. It is assumed here that the structure is field-aligned. The slab is thus separated from the surrounding environment by tangential discontinuity (TD) interfaces. The present paper aims at describing the self-consistent steady-state solution of such a configuration. A similar problem has been addressed earlier by Echim et al. (2005). As with any tangential discontinuity structure, the role of the electric field perpendicular to the interfaces plays a fundamental role in determining the nature of the configuration. This is especially true when studying the effect of the cross-field plasma flow $\boldsymbol{V}_{0}$ through the slab and of the electrostatic potential difference $\Delta \phi_{0}$ between the slab and its environment.

A practical realisation of such a plasma configuration may be found in the substorm magnetosphere when plasma is injected into the inner magnetosphere in a localised magnetic local time region, as sketched in Fig. 1. This injected plasma will extend along the magnetic field lines, and if the injection is maintained long enough, a slab can be formed with hot plasma that flows Earthward with respect to the colder plasmatrough environment (see, e.g., Zhang et al., 2008). Because of the importance of this particular problem, the present paper focuses on a slab of hot plasma of plasma sheet origin embedded in a colder plasmatrough background.

The paper is organised as follows. Section 2 presents a fully kinetic self-consistent model of the slab problem. The basic configuration is discussed in Sect. 3. In Sect. 4 the effect of the cross-field flow is examined. Particular attention is paid to the existence of limits to the flow speed for which an equilibrium solution to the problem can be found. Section 5 looks at the effect of the potential difference between the slab and its environment in situations without and with cross-field flow. The paper concludes with a discussion of the merits and limitations of the model and indicates its relevance for other magnetospheric phenomena.

\section{Kinetic TD model}

Let the slab be a quasi-steady structure that is exactly fieldaligned (Fig. 2). The $x$ axis is perpendicular to the slab, $z$ points along the magnetic field $\boldsymbol{B}_{0}$ at the centre of the slab, and the $y$ axis completes the right-hand reference frame. The magnetic field may rotate in the $y z$ plane. The cross-field 


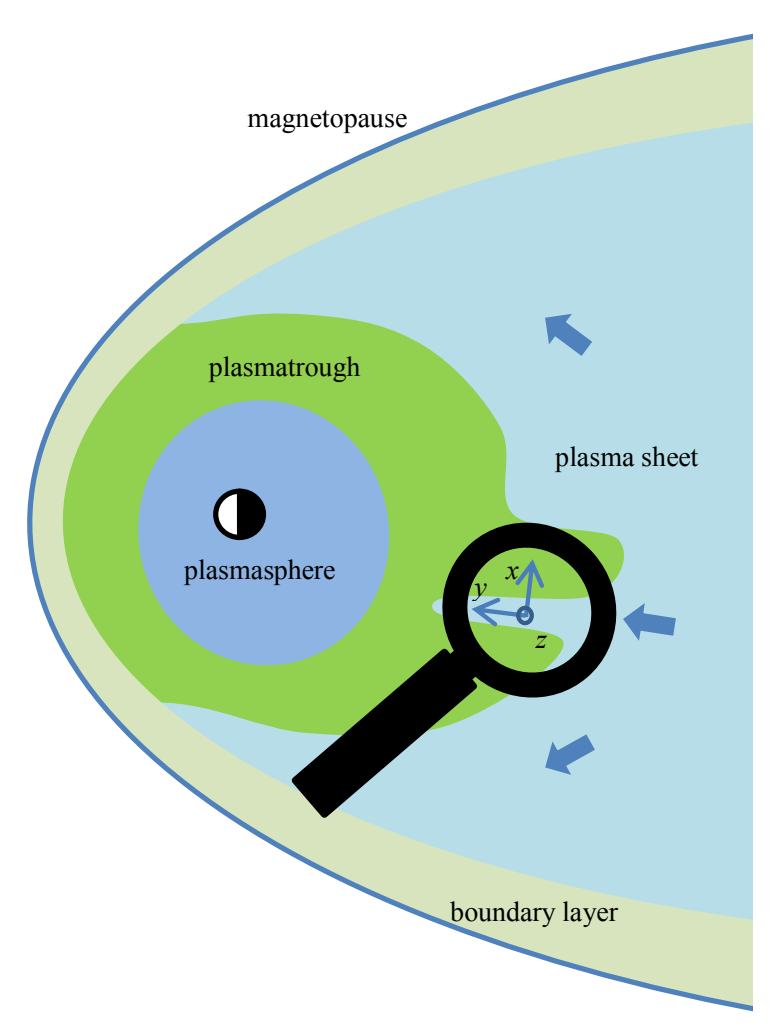

Fig. 1. Equatorial cross-section of the magnetosphere. Plasma injected from the plasma sheet can penetrate into the plasmatrough, thereby forming a cross-field flow channel. The magnifying glass zooms in on such a channel, which can be approximated by a 1dimensional plasma slab geometry.

flow $\boldsymbol{V}_{0}$ can have any orientation, but in most situations studied here it is considered to be exactly perpendicular to $\boldsymbol{B}_{0}$, along the $y$ axis, in which case the magnetic field direction does not change. The background is stationary.

\subsection{Velocity distribution functions}

The structure of TD interfaces has been studied in detail by Harris (1962), Nicholson (1963), Sestero (1964), Sestero (1966), Whipple et al. (1984), Roth et al. (1996), Mottez (2003), De Keyser and Echim (2013) and others. The guiding centre of each particle in a one-dimensional planar TD stays at a constant distance from the interface. How the particles are distributed in the system is, to a large extent, arbitrary. Only consideration of the "accessibility problem" (Whipple et al., 1984) can resolve this question: it requires studying the time evolution of the system, or a higher-dimensional version of the problem, in order to find out how particles enter into the TD layer. Nevertheless, a realistic set of particle velocity distributions can be put forward based on only a few parameters. The Vlasov-Maxwell equations must then be solved, where the Vlasov equations express the conservation of particles in phase space and Maxwell's laws impose constraints on the allowed plasma and field configurations, essentially

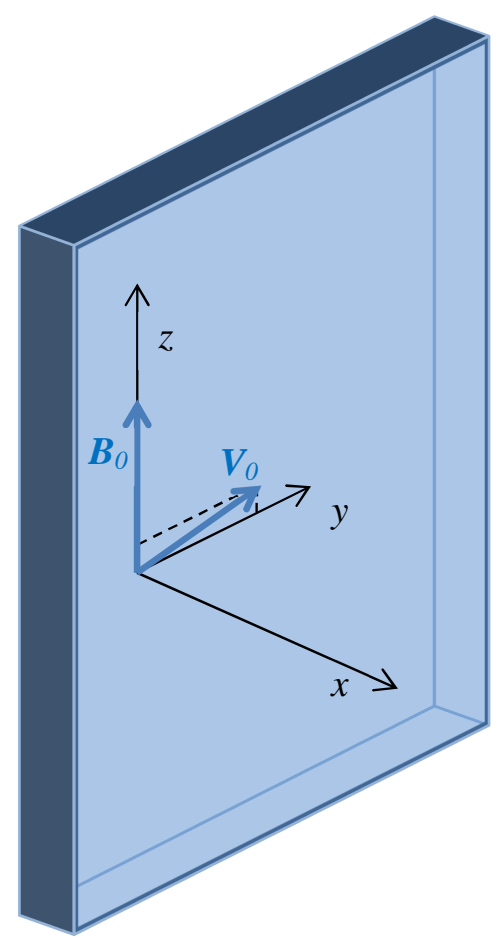

Fig. 2. Sketch of the slab configuration. A slab of plasma is embedded in a uniform background. The reference frame is chosen so that $x$ is normal to the structure and the magnetic field $\boldsymbol{B}_{0}$ points along the $z$ axis at the centre of the slab; the $y$ axis completes the righthanded frame. The flow $\boldsymbol{V}_{0}$ in the slab may have any orientation, although most often it is taken here along the $y$ direction.

total pressure balance. Another way to solve the accessibility problem is by including a diffusion process, or a (small) normal magnetic field component (e.g. Artemyev, 2011), but then one is altering the nature of the plasma boundary.

We use a slight generalisation of the planar TD model of Roth et al. (1996). All physical quantities vary only in the normal direction (the $x$ axis). The constants of motion of a particle of species $s$ with charge $Z_{s} e$ and mass $m_{s}$ in a TD configuration are its energy and its canonical momenta

$$
\begin{aligned}
H & =\frac{1}{2} m_{s} v^{2}+Z_{s} e \phi, \\
p_{y} & =m_{s} v_{y}+Z_{s} e A_{y}, \\
p_{z} & =m_{s} v_{z}+Z_{s} e A_{z} .
\end{aligned}
$$

The electromagnetic fields are represented by the scalar electric potential $\phi(x)$ and the magnetic vector potential $\boldsymbol{A}=$ $\left[0, A_{y}(x), A_{z}(x)\right]$. Particles in such a TD configuration may experience an electric and/or gradient-B drift parallel to the plane of the TD, but their guiding centre remains at constant $x$. In that sense, all particles in a static TD are "trapped". For given plasma and field states on either side of a TD, infinitely many configurations are possible as long as they satisfy the pressure balance condition. Kinetic TD models such as the one of Roth et al. (1996) choose "reasonable" particle 
distributions to specify the plasma arrangement. This choice results in TD descriptions that are quite realistic, as demonstrated by a number of studies that match model and observations (e.g. De Keyser et al., 1996; Hubert et al., 1998; Echim et al., 2009, 2011). We will come back to this choice in the discussion section.

Let $f_{s}\left(H, p_{y}, p_{z}\right)$ be the velocity distribution function (VDF) of population $s$. This VDF is written as the product of a Maxwellian function $F_{s}\left(H, p_{y}, p_{z}\right)$ and a smooth "cutoff function" $C_{s}\left(p_{y}, p_{z}\right)$, so that

$$
f_{s}\left(H, p_{y}, p_{z}\right)=C_{s}\left(p_{y}, p_{z}\right) F_{s}\left(H, p_{y}, p_{z}\right) .
$$

The Maxwellian distribution for particles with mass $m_{s}$, charge number $Z_{s}$, temperature $\mathcal{T}_{s}$, mean velocity $\mathcal{V}_{\mathrm{s}}$, and density normalisation constant $\mathcal{N}_{s}$, can be expressed as

$$
F_{S}\left(H, p_{y}, p_{z}\right)=\mathcal{N}_{s}\left(\frac{m_{s}}{2 \pi \kappa_{s}}\right)^{\frac{3}{2}} \mathrm{e}^{-\left(H+\frac{m_{s} V_{s}^{2}}{2}-p_{y} \mathcal{V}_{s y}-p_{z} \mathcal{V}_{s z}\right) / \kappa_{(}(5)}
$$

in terms of the invariants of motion, where $\kappa_{s}=k_{\mathrm{B}} \mathcal{T}_{s}$ denotes the characteristic thermal energy. The cutoff function is a smooth step-wise transition (a generalisation of Sestero, 1964, 1966; Lee and Kan, 1979). It is expressed in terms of the constants of motion as

$$
\begin{aligned}
C_{s}\left(p_{y}, p_{z}\right)= & v_{s}^{(1)} \mathcal{C}_{s}\left(-p_{z^{\prime}} ;-s_{s} p_{0 s}^{(1)},-\mathcal{V}_{s z^{\prime}}, \ell_{s}^{(1)}\right) \\
& +v_{s}^{(r)} \mathcal{C}_{s}\left(p_{z^{\prime}} ; s_{s} p_{0 s}^{(r)}, \mathcal{V}_{s z^{\prime}}, \ell_{s}^{(r)}\right)-v_{s}^{(c)}
\end{aligned}
$$

for reasons that are clarified below. Here, $s_{s}=\operatorname{sign} Z_{s}$, and $v_{s}^{(\mathrm{l})}, v_{s}^{(r)}, v_{s}^{(c)}, p_{0 s}^{(\mathrm{l})}, p_{0 s}^{(r)}, \ell_{s}^{(\mathrm{l})}$, and $\ell_{s}^{(r)}$ are parameters, while $p_{z^{\prime}}=-p_{y} \sin \theta_{s}+p_{z} \cos \theta_{s}$ and $\mathcal{V}_{s z^{\prime}}=-\boldsymbol{V}_{\text {sy }} \sin \theta_{s}+$ $\boldsymbol{V}_{\mathrm{s} z} \cos \theta_{s}$ are the $z$ coordinates of momentum and velocity in a frame that is rotated over an angle $\theta_{s}$, in between the asymptotic magnetic field clock angles $\theta_{\text {left }}$ and $\theta_{\text {right }}$ so that $B_{y^{\prime}}>0$ far from the TD (assuming that the magnetic field rotation is less than $180^{\circ}$ ). The function $\mathcal{C}_{s}$,

$\mathcal{C}_{s}\left(p_{z^{\prime}} ; s_{s} p_{0 s}, \mathcal{V}_{s z^{\prime}}, \ell_{s}\right)=\frac{1}{2} \operatorname{erfc} \frac{p_{z^{\prime}}-m_{s} \mathcal{V}_{s z^{\prime}}-s_{s} p_{0 s}}{s_{s} \sqrt{2 m_{s} \kappa_{s}\left(\ell_{s}^{2}-1\right)}}$,

where "erfc" denotes the complementary error function, switches from 1 when $p_{z^{\prime}} \ll m_{s} \mathcal{V}_{s z^{\prime}}+s_{s} p_{0 s}$ to 0 for $p_{z^{\prime}} \gg$ $m_{s} \mathcal{V}_{s z^{\prime}}+s_{s} p_{0 s}$ for ions, and the reverse for electrons. As $A_{z^{\prime}}=-\int B_{y^{\prime}} d x>0$ for $x \rightarrow-\infty$ and $<0$ for $x \rightarrow+\infty$, this switching function is $\mathcal{C}_{s}=0$ at $x=-\infty$ and $\mathcal{C}_{s}=1$ at $x=+\infty$ for both ions and electrons. The parameter $\ell_{s} \geq 1$ determines the momentum range over which the switch occurs, i.e. the smoothness of the transition, while $p_{0 s}$ controls the position of the transition in momentum space. The choice of the cutoff function $C_{S}$ allows the following possibilities:

- Type I: $v_{s}^{(1)}=1, v_{s}^{(r)}=0, v_{s}^{(c)}=0$. In this case $C_{s}=1$ at $x=-\infty$ and $C_{s}=0$ at $x=+\infty$ so that the VDF is Maxwellian at $x=-\infty$ but vanishes at $x=+\infty$. The population is therefore confined to the left half-space. The cutoff is centred around $m_{s} \mathcal{V}_{s z^{\prime}}+s_{s} p_{0 s}^{(1)}$ and has a dimensionless width $\ell_{s}^{(1)}$. This Type I cutoff is used to represent the stationary background populations in the left half-space.

- Type II: $v_{s}^{(1)}=0, v_{s}^{(r)}=1, v_{s}^{(c)}=0$. In this case $C_{s}=$ 1 at $x=+\infty$ and $C_{s}=0$ at $x=-\infty$. The VDF is Maxwellian at $x=+\infty$ and vanishes at $x=-\infty$ : the population is confined to the right half-space, with cutoff position $m_{s} \mathcal{V}_{s z^{\prime}}+s_{s} p_{0 s}^{(r)}$ and dimensionless width $\ell_{s}^{(r)}$. This Type II cutoff can represent the stationary background populations in the right half-space.

- Type III: $v_{s}^{(1)}=1, v_{s}^{(r)}=1, v_{s}^{(c)}=0$, with $p_{0 s}^{(1)}=p_{0 s}^{(r)}$ and $\ell_{s}^{(1)}=\ell_{s}^{(r)}$. In this case $C_{s} \equiv 1$ so that the distribution is Maxwellian throughout the domain. The population then is not confined to a particular subspace by the cutoff function, but it may still be constrained by the electric or magnetic forces. A typical example is the ion and electron populations in a Harris current sheet, which are both confined to the current sheet region via their diamagnetic drift velocities (Harris, 1962). This type of cutoff will not be used in the slab model presented here.

- Type IV: $v_{s}^{(1)}=1, v_{s}^{(r)}=1, v_{s}^{(c)}=1$, with $p_{0 s}^{(1)}<p_{0 s}^{(r)}$. In this case the population is confined to an interval, between boundaries determined by $p_{0 s}^{(1)}$ and $p_{0 s}^{(r)}$, with dimensionless widths $\ell_{s}^{(1)}$ and $\ell_{s}^{(r)}$. The slab populations will be represented using this type of cutoff function.

These different choices for $C_{s}$ are depicted in Fig. 3. It is now possible to construct a discontinuity model in which the plasma arrangement is expressed as a combination of species with VDFs that have this parameterised form.

The use of localised populations with a two-sided cutoff is a hallmark of the present study. Such two-sided cutoffs have been introduced earlier by Echim et al. (2005); the formalism used there relies on a description in terms of the invariants $H$, $p_{y}$, and $\mu$, where the magnetic moment $\mu$ is an approximate adiabatic invariant that can be used when the magnetic field changes along $z$ in a 2-dimensional configuration. The cutoff function used by these authors is a Heaviside function, which is the limit case for $\ell \rightarrow 1$. The introduction of electron distribution functions with cutoffs $\ell_{-}=1$ gives rise to very localised electron transition layers with correspondingly large local electric fields that are likely to produce instabilities, which tend to widen such layers. The added flexibility brought by introducing the characteristic length scales therefore increases the realism of the solutions.

An interesting property of this particular form of VDF is that its moments

$$
Q_{s}^{(i j k)}=\iiint v_{x}^{i} v_{y}^{j} v_{z}^{k} f_{s}\left(v_{x}, v_{y}, v_{z}\right) \mathrm{d} v_{x} \mathrm{~d} v_{y} \mathrm{~d} v_{z}
$$

can be computed analytically so that the partial densities $n_{s}=Q_{s}^{(000)}$ and currents $j_{s y}=Z_{s} e Q_{s}^{(010)}$ and $j_{s z}=$ 


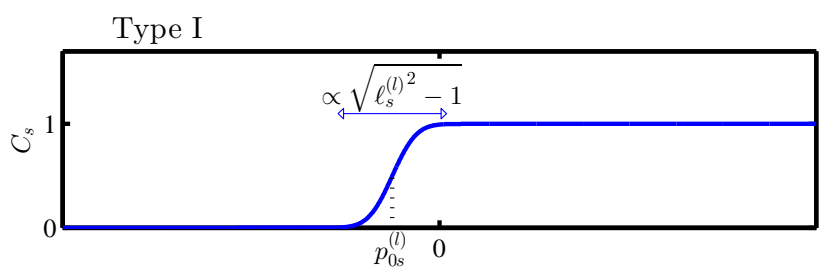

Type II

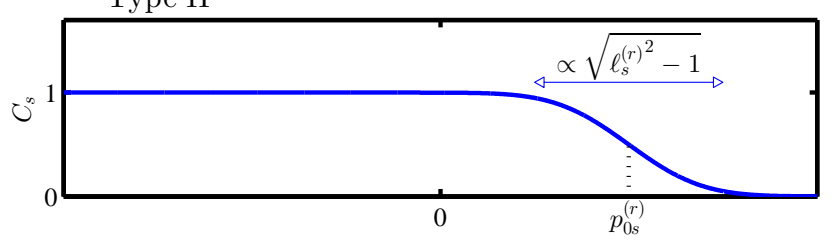

Type III

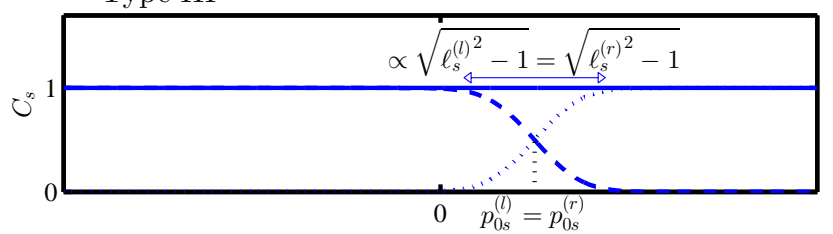

Type IV

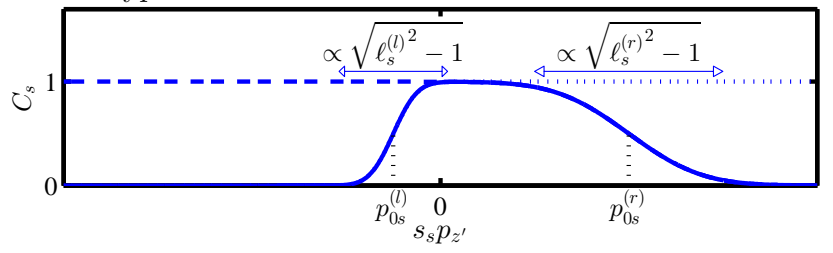

Fig. 3. Four types of cutoff functions are considered: Type I and II produce populations that are constrained to one side of the transition; Type III corresponds to Maxwellian distributions, and Type IV is a two-sided cutoff function. Note that $s_{s} p_{z^{\prime}} \sim|Z| e A_{z^{\prime}}$ corresponds to $+\infty$ if $x \rightarrow-\infty$ and to $-\infty$ if $x \rightarrow+\infty$.

$Z_{S} e Q_{s}^{(001)}$ are expressed in terms of the potentials $\phi$ and $\boldsymbol{A}$. Appendix A gives the derivation of these moments.

\subsection{Differential equations and boundary conditions}

The time-stationary Maxwell equations can be formulated as

$$
\begin{aligned}
\frac{\mathrm{d} A_{y}}{\mathrm{~d} x} & =+B_{z}(x), \\
\frac{\mathrm{d} A_{z}}{\mathrm{~d} x} & =-B_{y}(x), \\
\frac{\mathrm{d} B_{y}}{\mathrm{~d} x} & =+\mu_{0} \sum_{s} j_{s z}\left(\phi(x), A_{y}(x), A_{z}(x)\right), \\
\frac{\mathrm{d} B_{z}}{\mathrm{~d} x} & =-\mu_{0} \sum_{s} j_{s y}\left(\phi(x), A_{y}(x), A_{z}(x)\right), \\
0 & =\sum_{s} Z_{s} n_{s}\left(\phi(x), A_{y}(x), A_{z}(x)\right) .
\end{aligned}
$$

The last equation is the quasi-neutrality condition; it replaces the Poisson equation. This nonlinear differential algebraic set of equations is solved numerically, yielding the electromag- netic potentials $\phi$ and $\boldsymbol{A}$. The steady-state solution of the TD configuration has then been found, since all VDFs are defined in terms of the invariants of motion, and these invariants are given in terms of $\phi$ and $\boldsymbol{A}$. Note that the solutions proposed by Harris (1962), Nicholson (1963), and Mottez (2003) do not require the quasi-neutrality or Poisson equation, since they are exactly charge neutral by construction, which is a severe restriction.

This set of equations has to be supplemented with boundary conditions. We assume that the magnetic field $\boldsymbol{B}_{0}$ is given at a reference point $x_{0}$. The electric and magnetic potentials can be set to zero there, $\phi\left(x_{0}\right)=0$ and $\boldsymbol{A}\left(x_{0}\right)=\mathbf{0}$, since they are determined only up to a constant. In all calculations presented here, the choice $x_{0}=0$ is made. The magnetic field is taken to be exactly along $z$ at $x_{0}$. Using the expressions for plasma density obtained in Appendix A, and always considering that the background populations are stationary $\left(\mathcal{V}_{\mathrm{s}}=\mathbf{0}\right)$, the density of the background populations of Type I at $-\infty$ is

$n_{S}(-\infty)=\mathcal{N}_{S} \exp \left(-\frac{Z_{s} e}{\kappa_{S}} \phi(-\infty)\right) ;$

knowing $n_{s}(-\infty)$, it is therefore possible to compute $\mathcal{N}_{s}$ if $\phi(-\infty)$ is given as a boundary condition. For the background populations of Type II the density is specified at $+\infty$, and the density normalisation constant is obtained from

$n_{s}(+\infty)=\mathcal{N}_{s} \exp \left(-\frac{Z_{s} e}{\kappa_{s}} \phi(+\infty)\right)$.

For Type III populations (which are not used here) the density can be specified at $x_{0}=0$, with the normalisation constant following from

$n_{s}(0)=\mathcal{N}_{s}$.

In the present paper we will limit ourselves to configurations in which the Type IV slab populations do not vanish at $x_{0}=$ 0 , so that their density can be specified there as well. One obtains

$n_{s}(0)=\mathcal{N}_{s}\left[\frac{1}{2} \operatorname{erfc} \frac{p_{0 s}^{(1)}}{\ell_{s}^{(1)} \sqrt{2 m_{s} \kappa_{s}}}+\frac{1}{2} \operatorname{erfc} \frac{-p_{0 s}^{(r)}}{\ell_{s}^{(r)} \sqrt{2 m_{s} \kappa_{s}}}-1\right] ;$

note that $n_{s}(0)=\mathcal{N}_{S}$ when $x_{0}$ is fully embedded in the slab and if the slab is wide enough, since the distribution then is fully Maxwellian there $\left(p_{0 s}^{(1)} \ll 0 \ll p_{0 s}^{(r)}\right)$. In summary, after specifying $\phi(-\infty)$ and $\phi(+\infty)$ one can integrate the differential-algebraic problem from $x_{0}$ down to $-\infty$ and from $x_{0}$ up to $+\infty$.

\section{Basic configuration}

By means of example, we consider the case of hot plasma sheet material injected into the colder plasmatrough. The 


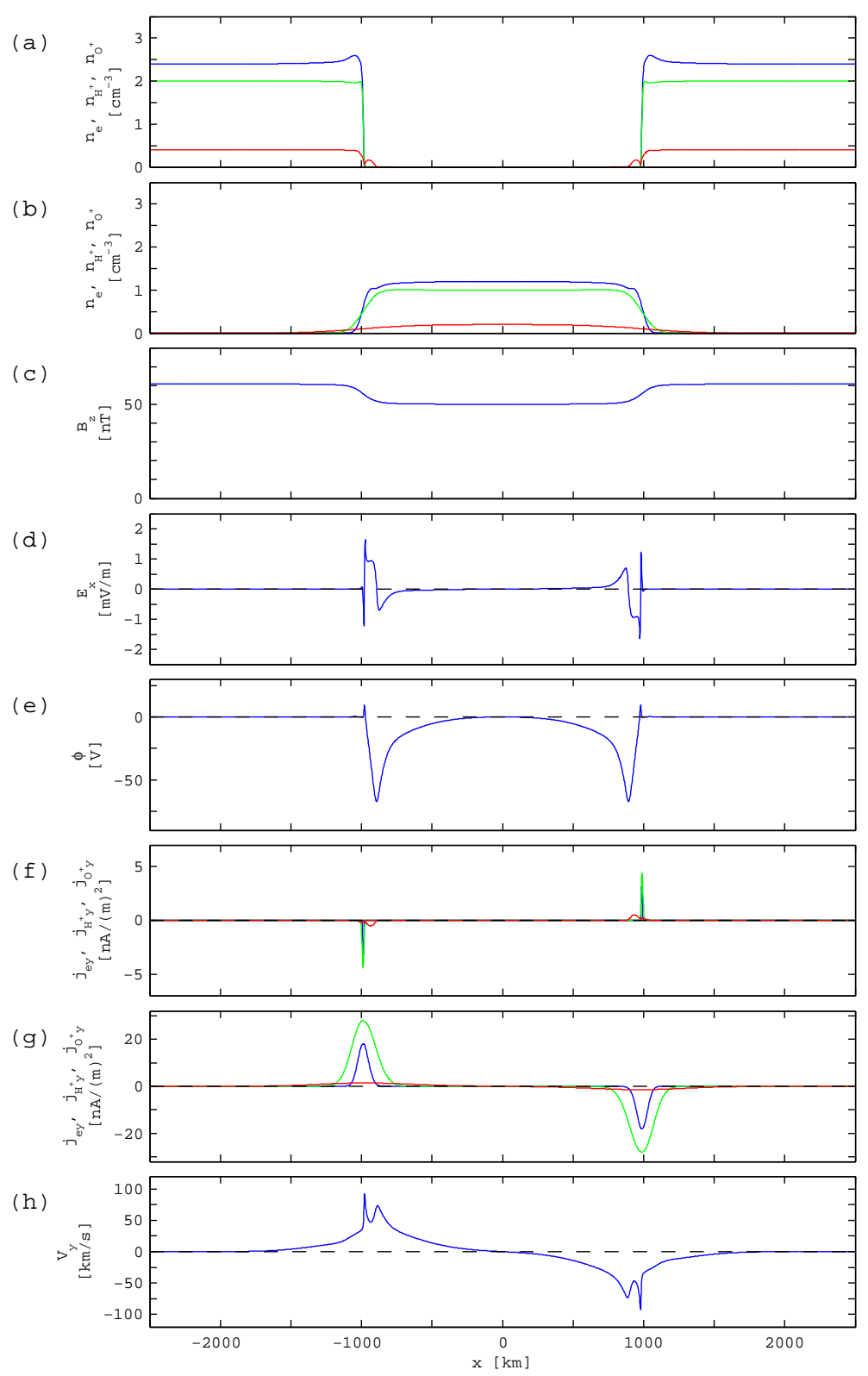

Fig. 4. Plasma and field structure of a $2000 \mathrm{~km}$-wide plasma slab embedded in a uniform background. The plasma parameters are given in Table 1 . There is no cross-field flow at the slab centre, $V_{0 y}=0$, and the electric potential boundary conditions are zero, $\phi( \pm \infty)=0$. The panels show (a) the densities of the background populations, blue for the electrons, green for the $\mathrm{H}^{+}$ions, red for the $\mathrm{O}^{+}$ions; (b) the density profiles of the slab populations, blue for the electrons, green for $\mathrm{H}^{+}$, red for $\mathrm{O}^{+}$; (c) the magnetic field magnitude; (d) the electric field; (e) the electric potential; (f) the partial currents along $y$ of the background populations, with the same colour code as before; (g) the partial currents along $y$ of the slab populations; and (h) the plasma bulk velocity along $y$.

plasmatrough plasma in reality consists of several populations (Reasoner et al., 1983; Sojka et al., 1983), but to simplify matters it is taken here to be a plasma consisting of $3 \mathrm{eV}$ Maxwellian electrons, $12 \mathrm{eV}$ protons, and $12 \mathrm{eV}$ oxygen ions. The plasma sheet particle energies often vary, but they are much larger than the plasmatrough ones; typical values are $0.5 \mathrm{keV}$ for the electrons, and $2 \mathrm{keV}$ for the protons and oxygen ions. The plasmatrough plasma is taken to be twice as dense as the hot plasma sheet material. The injection speeds can be significant. While the speed of bursty flows in the magnetotail may be up to hundreds of $\mathrm{km} \mathrm{s}^{-1}$ (Angelopoulos et al., 1992), flow braking must occur as this material approaches the near-Earth magnetosphere (e.g. Deng et al., 2005). Beams in the plasma sheet boundary are essentially field-aligned, while the bulk flow in the braking region is essentially perpendicular to the field. A range of speeds will be 
Table 1. Typical particle properties for a plasmatrough background and a plasma sheet slab: density $n$, temperature $T$, gyroradius $\rho$ in a $50 \mathrm{nT}$ field, and thermal velocity $v_{\text {th }}$, as well as the cutoff function parameters $\ell^{(\mathrm{l})}, \ell^{(\mathrm{r})}, p_{0}^{(\mathrm{l})}$ and $p_{0}^{(\mathrm{r})}$.

\begin{tabular}{rrrrrrrrrr}
\hline Species & $n\left[\mathrm{~cm}^{-3}\right]$ & $T[\mathrm{eV}]$ & $\rho[\mathrm{km}]$ & $\ell^{(1)}$ & $\ell^{(\mathrm{r})}$ & $p_{0}^{(1)}\left[\mathrm{kg} \mathrm{m} \mathrm{s}^{-1}\right]$ & $p_{0}^{(\mathrm{r})}\left[\mathrm{kg} \mathrm{m} \mathrm{s}^{-1}\right]$ & $\ell \rho\left[\mathrm{km}^{2}\right.$ & $v_{\mathrm{th}}\left[\mathrm{km} \mathrm{s}^{-1}\right]$ \\
\hline Left $^{-}$ & 2.4 & 3 & 0.12 & 40 & & $8 \times 10^{-21}$ & & 4.7 & 1028 \\
$\mathrm{H}^{+}$ & 2.0 & 12 & 9.80 & 1 & & $8 \times 10^{-21}$ & & 9.8 & 49 \\
$\mathrm{O}^{+}$ & 0.4 & 12 & 39.2 & 1 & & $8 \times 10^{-21}$ & & 39.2 & 12 \\
\hline Right $^{-}$ & 2.4 & 3 & 0.12 & & 40 & & $-8 \times 10^{-21}$ & 4.7 & 1028 \\
$\mathrm{H}^{+}$ & 2.0 & 12 & 9.80 & & 1 & & $-8 \times 10^{-21}$ & 9.8 & 49 \\
$\mathrm{O}^{+}$ & 0.4 & 12 & 39.2 & & 1 & & $-8 \times 10^{-21}$ & 39.2 & 12 \\
\hline Slab e $^{-}$ & 1.2 & 500 & 1.51 & 40 & 40 & $-8 \times 10^{-21}$ & $8 \times 10^{-21}$ & 60.4 & 13268 \\
$\mathrm{H}^{+}$ & 1.0 & 2000 & 127 & 1 & 1 & $-8 \times 10^{-21}$ & $8 \times 10^{-21}$ & 127 & 633 \\
$\mathrm{O}^{+}$ & 0.2 & 2000 & 506 & 1 & 1 & $-8 \times 10^{-21}$ & $8 \times 10^{-21}$ & 506 & 158 \\
\hline
\end{tabular}

examined here with $V_{0}$ on the order of some $10 \mathrm{kms}^{-1}$. The magnetic field is taken to be $50 \mathrm{nT}$ at $x_{0}=0$. In addition, there can be nonzero electric potential differences $\phi(-\infty)$ and $\phi(+\infty)$. Table 1 summarises the properties of the plasma populations that are involved. Note that the dimensionless length scales of the TD transitions are taken to be $\ell_{s}=1$ for the ions and $\ell_{s}=40$ for the electrons: otherwise too strong charge separation electric fields would arise that would immediately lead to instabilities and a corresponding widening of the layer. The resulting characteristic spatial lengths in the solution, $\ell_{s} \rho_{s}$, still differ by up to two orders of magnitude, which makes the use of a variable step integrator a must.

Figure 4 shows the configuration obtained without crossfield flow, $V_{0}=0$, and without net electric potential differences across the TD interfaces, $\phi( \pm \infty)=0$, for a slab consisting of plasma sheet ions and electrons (Type IV populations) embedded in a plasmatrough ion and electron background on either side (Type I and Type II distributions). The slab is $2000 \mathrm{~km}$ wide. This particular width is obtained by choosing an appropriate value for parameter $p_{0}= \pm 8 \times$ $10^{-21} \mathrm{~kg} \mathrm{~ms}^{-1}$ for all the cutoffs (for the signs, see Table 1). The slab width is taken larger than the plasma sheet oxygen gyroradius to obtain distinct TD boundaries on either side of the slab. The background and slab particle density profiles in Fig. $4 \mathrm{a}$ and $\mathrm{b}$ are symmetric. In the present configuration the magnetic field direction does not change; only its magnitude varies a little (Fig. 4c) as plasma $\beta=0.06$ at $x_{0}$ is low. The electric field inside each of the TD interfaces separating the slab from the background is on the order of $1 \mathrm{mVm}^{-1}$ (Fig. 4d). The electric field profile in each TD has a complex nature, with small spatial scales on the outside and broader variations inside the slab; this is due to the difference in gyroradii. It is not surprising that the electric field, and hence also the electric potential (Fig. 4e), changes on spatial scales of hundreds of kilometres inside the slab: these are the plasma sheet ion gyroradius scales. Figures $4 \mathrm{f}$ and $4 \mathrm{~g}$ show the partial currents carried by each of the populations. The currents are all along $y$. It is readily visible that the spa- tial thickness of the current-carrying layers is much larger for the slab populations than for the background populations, as the thicknesses scale with the gyroradii. The sense of the currents on either side of the slab is opposite. Figure $4 \mathrm{~h}$ shows that the bulk plasma velocity is not zero everywhere, even if $\mathcal{V}_{\mathrm{S}}=\mathbf{0}$ for all populations. The reason is that the VDFs are no longer Maxwellian inside the TDs: they lose their symmetry because of the cutoffs in the distribution functions. This can easily be understood: if the ions are confined to one halfspace with an abrupt cutoff, the ions penetrating the farthest into the other half-space will move in one predominant direction, corresponding to their gyrating motion. The electrons gyrate in the opposite sense, but because of their higher mass the ions dominate the plasma bulk velocity, which will therefore be nonzero. The flows of up to $80 \mathrm{~km} \mathrm{~s}^{-1}$ are localised at the TD interfaces, although the bulk flow remains nonzero for much of the slab interior. This is due to the large $\mathrm{O}^{+}$gyroradius and the fact that the $\mathrm{O}^{+}$ions, having 16 times the $\mathrm{H}^{+}$ mass, have a major contribution to the bulk flow. The bulk flow (essentially the ion flow) clearly has opposite directions in either interface.

There is no reason to assume that the plasma slab is at the same electric potential as its surroundings. If the above plasma configuration, still with $V_{0}=0$, is placed in an environment where $\phi( \pm \infty) \neq 0$, the internal structure of the TD interfaces is modified. Figure 5 shows such a situation where $\phi(-\infty)=50 \mathrm{~V}$ and $\phi(+\infty)=100 \mathrm{~V}$. The slab is then situated in an electric potential well. The net effect of these potential differences between the slab and the background plasma is to pull the slab electrons towards the exterior and to push the slab ions to the interior (compare Figs. $4 \mathrm{~b}$ and $5 b)$. This effect is small, since the additional charge separation created in this way immediately provokes a restoring polarisation electric field. For $\phi(-\infty)=\phi(+\infty)$ the configuration remains symmetric. An example is given in Fig. 6, where the potential well is quite deep with $\phi( \pm \infty)=500 \mathrm{~V}$.

Figure 7 shows the solution for an exactly perpendicular cross-field flow $V_{0 y}=10 \mathrm{~km} \mathrm{~s}^{-1}$; the magnetic field direction 


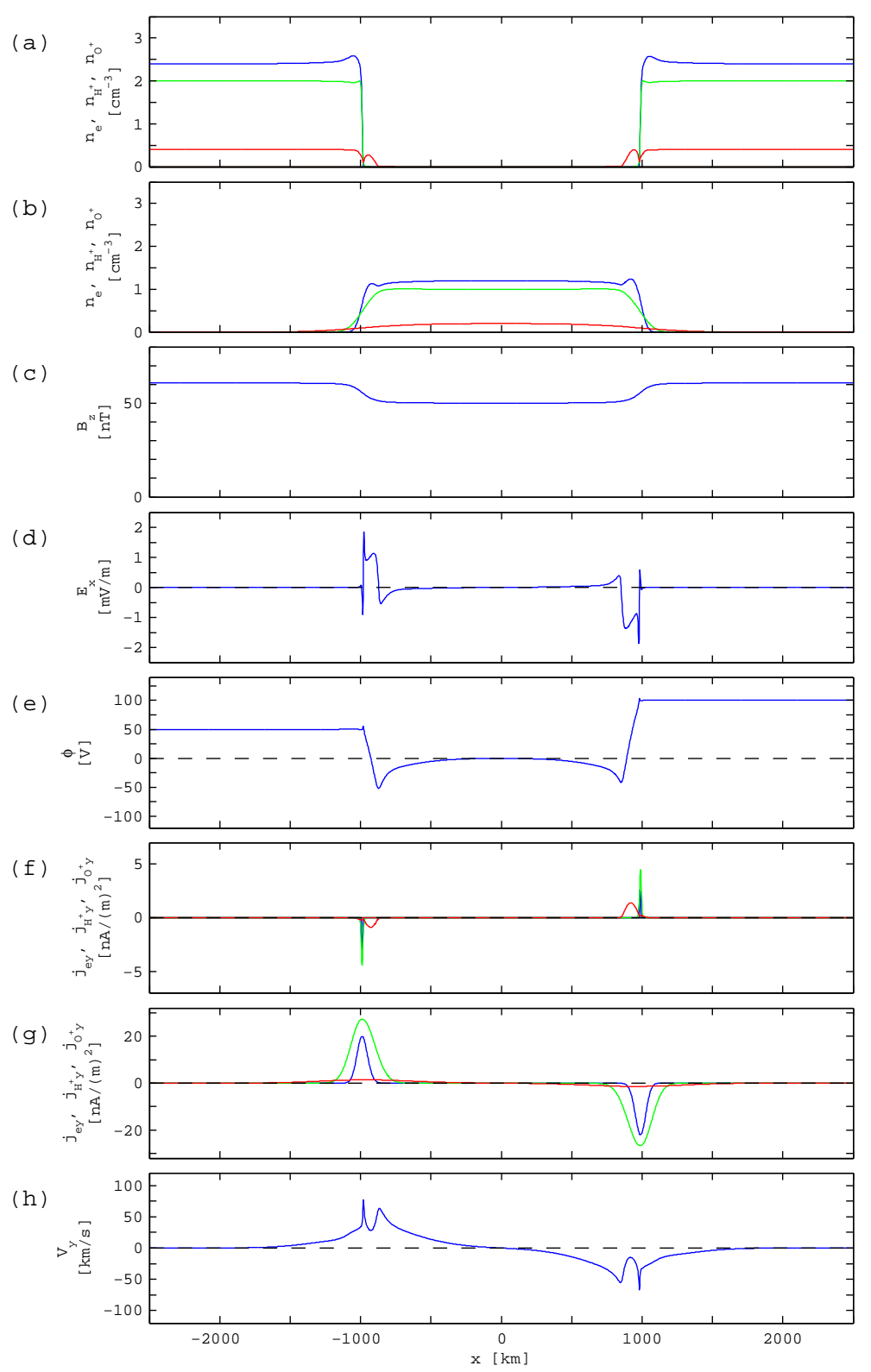

Fig. 5. Plasma and field structure of a $2000 \mathrm{~km}$-wide $\mathrm{e}^{-}-\mathrm{H}^{+}-\mathrm{O}^{+}$plasma slab embedded in a uniform background. The plasma parameters are given in Table 1 . There is no cross-field flow at the slab centre, $V_{0 y}=0$. The electric potential boundary conditions are $\phi(-\infty)=50 \mathrm{~V}$ and $\phi(+\infty)=100 \mathrm{~V}$. The figure format is the same as in Fig. 4.

then does not change. Such a flow produces a convection electric field in the slab of magnitude $E_{x}=-V_{0 y} B_{0} \approx$ $-0.5 \mathrm{mV} \mathrm{m}^{-1}$. Even though the flow is quite modest, due to the thickness of the slab the inner side of each TD interface is at a potential of $\pm 500 \mathrm{~V}$. The electric potential boundary conditions are $\phi( \pm \infty)=0 \mathrm{~V}$. This is similar to the problem studied by Echim et al. (2005). As these authors already noted, the configuration is not symmetric anymore as soon as $V_{0 y} \neq 0$, and reversing the sense of the flow will change the sense of the electric field inside each interface TD. Despite the bulk flow of $+10 \mathrm{~km} \mathrm{~s}^{-1}$ at the centre of the slab, strong deviations (even flows in the opposite sense) occur near the TD interfaces. An alternative configuration consists of considering $\phi( \pm \infty)= \pm 500 \mathrm{~V}$ to avoid strong potential differences across the two interfaces, as in Fig. 8.

In the above examples, the slab was taken wider than the plasma sheet $\mathrm{O}^{+}$gyroradius so that the slab is, in effect, sandwiched between two separate TD transitions, with the slab centre being characterised by uniform densities, magnetic field, electric field, and plasma velocity. Figure 9 shows that this does not necessarily have to be so. By choosing $p_{0}= \pm 2 \times 10^{-21} \mathrm{~kg} \mathrm{~m} \mathrm{~s}^{-1}$ for all the cutoffs, the slab width 


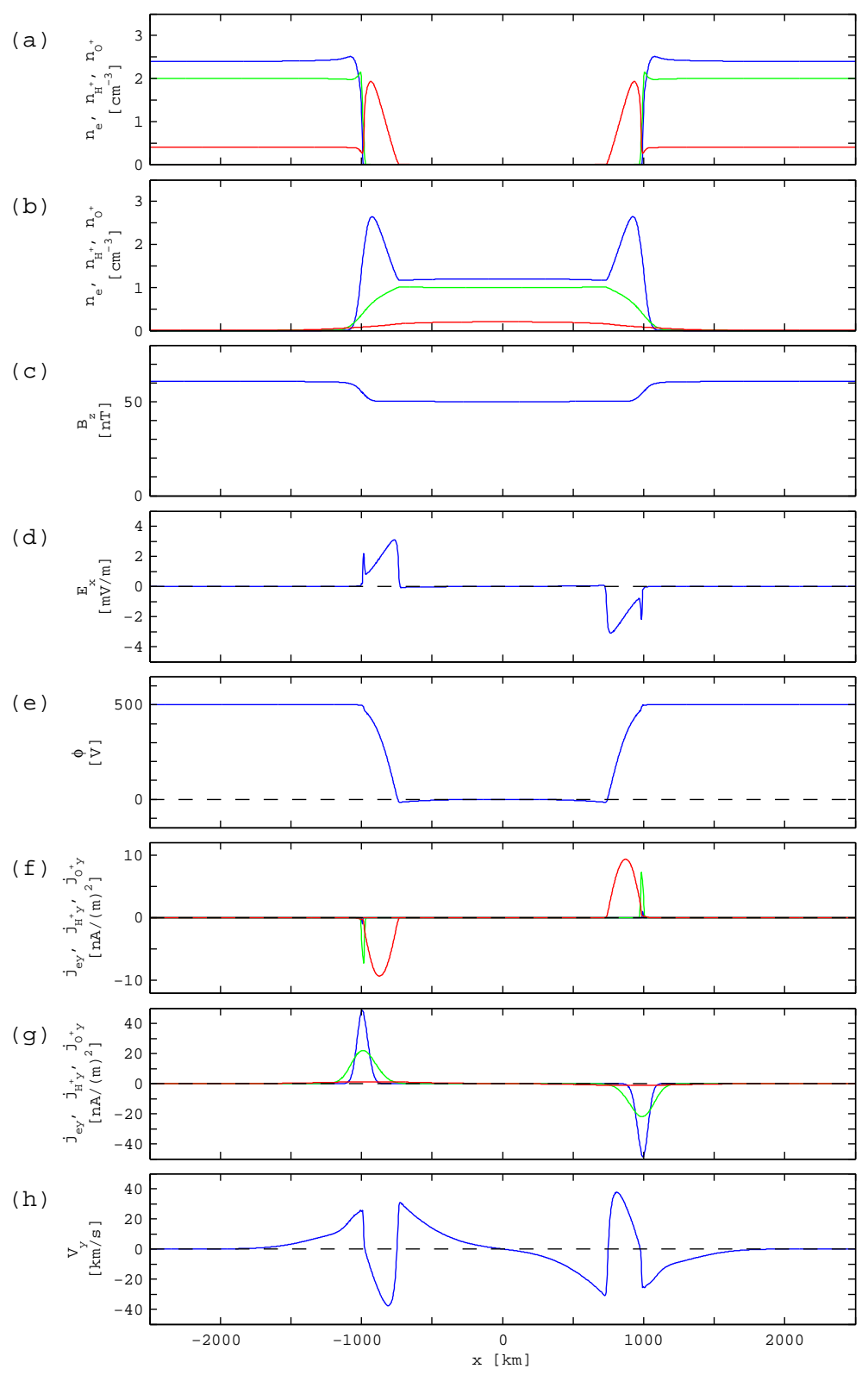

Fig. 6. Idem as Fig. 5 but now with $\phi( \pm \infty)=500 \mathrm{~V}$.

is reduced to $500 \mathrm{~km}$ so that the two TD transitions merge into one compound structure. All quantities show variations inside the slab.

\section{Cross-field flow}

We now examine the influence of the cross-field flow in more detail. To simplify matters, only proton-electron plasmas are considered; the plasma properties are still those of Table 1 for the $2000 \mathrm{~km}$-wide slab, but without $\mathrm{O}^{+}$ions, and with slab and background densities of 1 and $2 \mathrm{~cm}^{-3}$, respectively. First, consider the situation in which $\phi( \pm \infty)=0$. Figure 10 plots the minimum and maximum electric field strength in the slab while $V_{0 y}$ varies in steps of $2 \mathrm{~km} \mathrm{~s}^{-1}$ from -50 to $+50 \mathrm{~km} \mathrm{~s}^{-1}$. A first observation is that no equilibrium solution can be found that satisfies the imposed boundary conditions for $\left|V_{0 y}\right|>V_{0 \text {,Max }} \approx 35 \mathrm{~km} \mathrm{~s}^{-1}$. Because of the symmetry of the problem, it is obvious that the existence region is symmetric as well. This limit of existence for a slab is due to the fact that there is an intrinsic limit $\Delta \phi_{\max }$ to the potential difference that can be accommodated by an individual TD. This maximum potential difference is determined by the plasma properties on either side of the interface and by the transition lengths; in the present case this is about $3750 \mathrm{~V}$. In a slab of thickness $D$, the convection electric field 

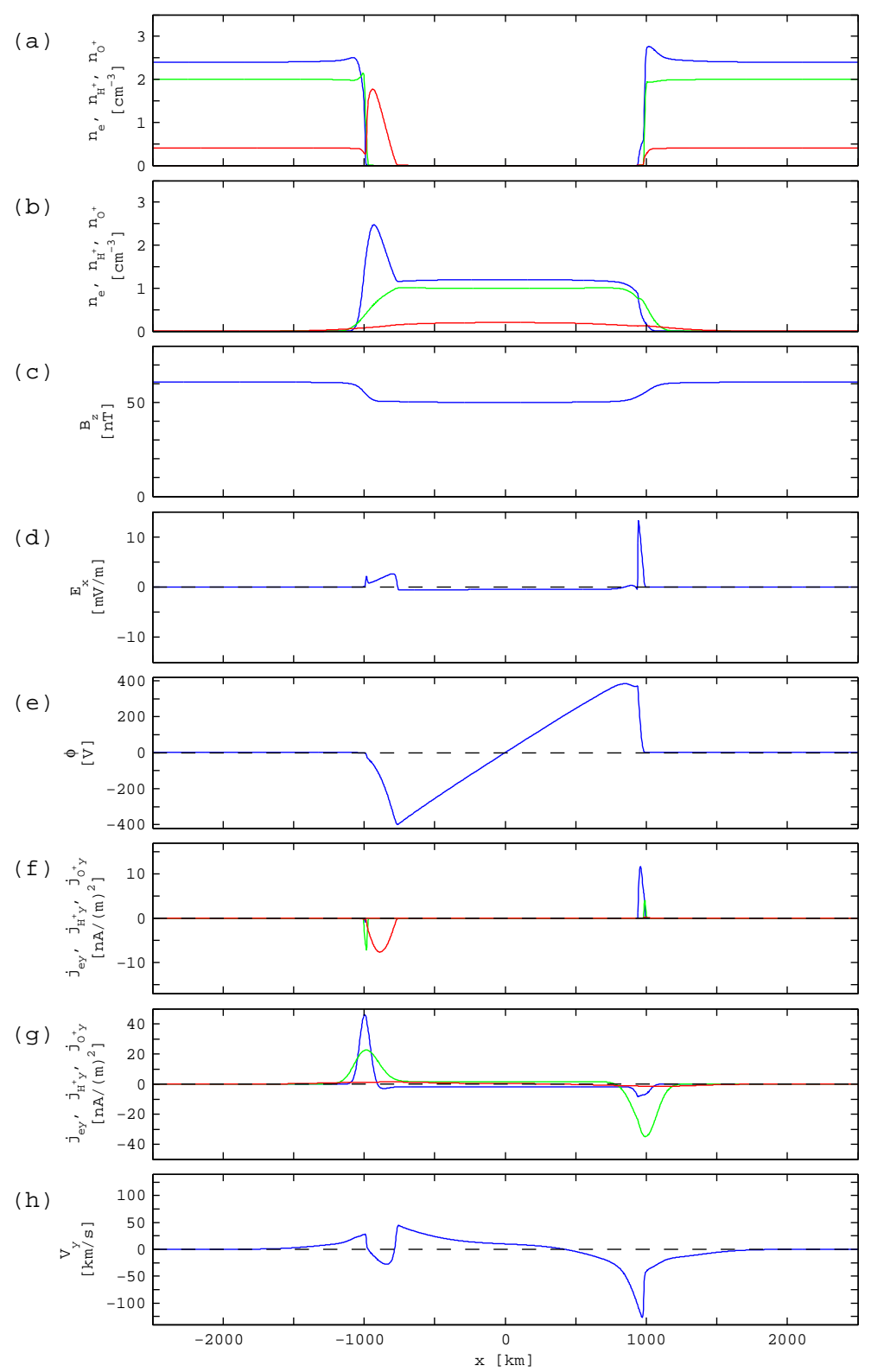

Fig. 7. Plasma and field structure of a $2000 \mathrm{~km}$-wide $\mathrm{e}^{-}-\mathrm{H}^{+}-\mathrm{O}^{+}$plasma slab embedded in a uniform background. The plasma parameters are given in Table 1 . There is a cross-field flow $V_{0 y}=10 \mathrm{~km} \mathrm{~s}^{-1}$ at the slab centre. The electric potential boundary conditions are $\phi( \pm \infty)=0 \mathrm{~V}$. The figure format is the same as in Fig. 4.

produced by the cross-field flow leads to an electric potential that reaches $\phi( \pm D / 2) \approx \pm V_{0 y} B_{0} D / 2$; if $\phi( \pm \infty)=0$, this results in a potential jump across the interface TDs. It is therefore clear that (at least for slabs that are broad enough so as to be considered as bounded by two separate TDs) the maximum cross-field flow that can be supported should vary inversely with slab width, i.e.

$\left|V_{0 y}\right|<V_{0, \max }=\frac{2 \Delta \phi_{\max }}{B_{0} D}$.
The question of the existence of an individual TD under shear flow conditions has been studied previously in considerable detail (Sestero, 1966; De Keyser et al., 1997; De Keyser and Echim, 2013). These studies have demonstrated that there is a distinct asymmetry in the size of the existence domain, depending on the sense of the flow shear. This asymmetry is caused by the interplay between the convection electric field related to the flow shear on the one hand, and the charge separation electric field due to the different gyroradii on the other hand. For one sense of the flow shear they strengthen each other, while they partially cancel for the opposite sense. For 


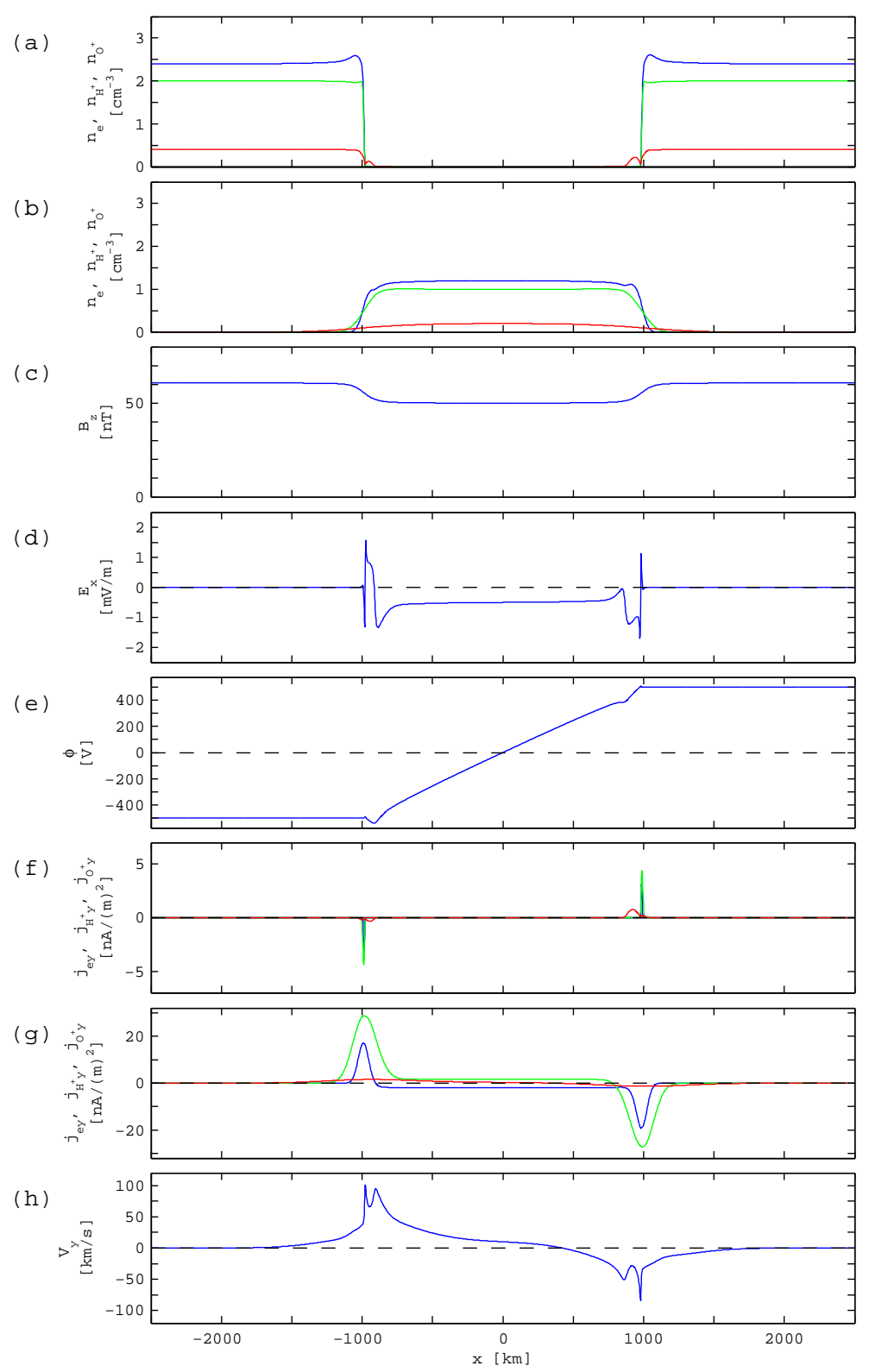

Fig. 8. Idem as Fig. 7 but now with $\phi( \pm \infty)= \pm 500 \mathrm{~V}$.

the slab configuration considered here, the sense of the flow shear at the two interfaces is opposite, so that the existence limit is always reached for pretty low cross-field flow at one of both TDs, whatever the sign of $V_{0 y}$.

It is, of course, also possible to consider a slab configuration where the electric potentials at either side match the potential difference due to the convection electric field in the slab, as we did earlier in Fig. 8. In that case the existence domain will be larger because the choice of the values $\phi( \pm \infty)$ reduces the electric potential differences across the TDs.

In general $\Delta \phi_{\max }$ increases with the temperature of the hot ions (see, e.g., De Keyser and Echim, 2013), so that the range of $V_{0 y}$ for which an equilibrium exists becomes correspondingly larger.

\section{External electric potential}

The existence of a slab equilibrium does not only depend on the flow shear, but also on the imposed electric potential difference $\Delta \phi_{0}$ between the centre of the slab and the background. This dependence is explored here by considering symmetric situations where $\Delta \phi_{0}=\phi( \pm \infty) \neq 0$, while $V_{0}=0 \mathrm{~km} \mathrm{~s}^{-1}$; the same proton-electron plasmas are considered as in the previous section. Figure 11 plots the minimum 


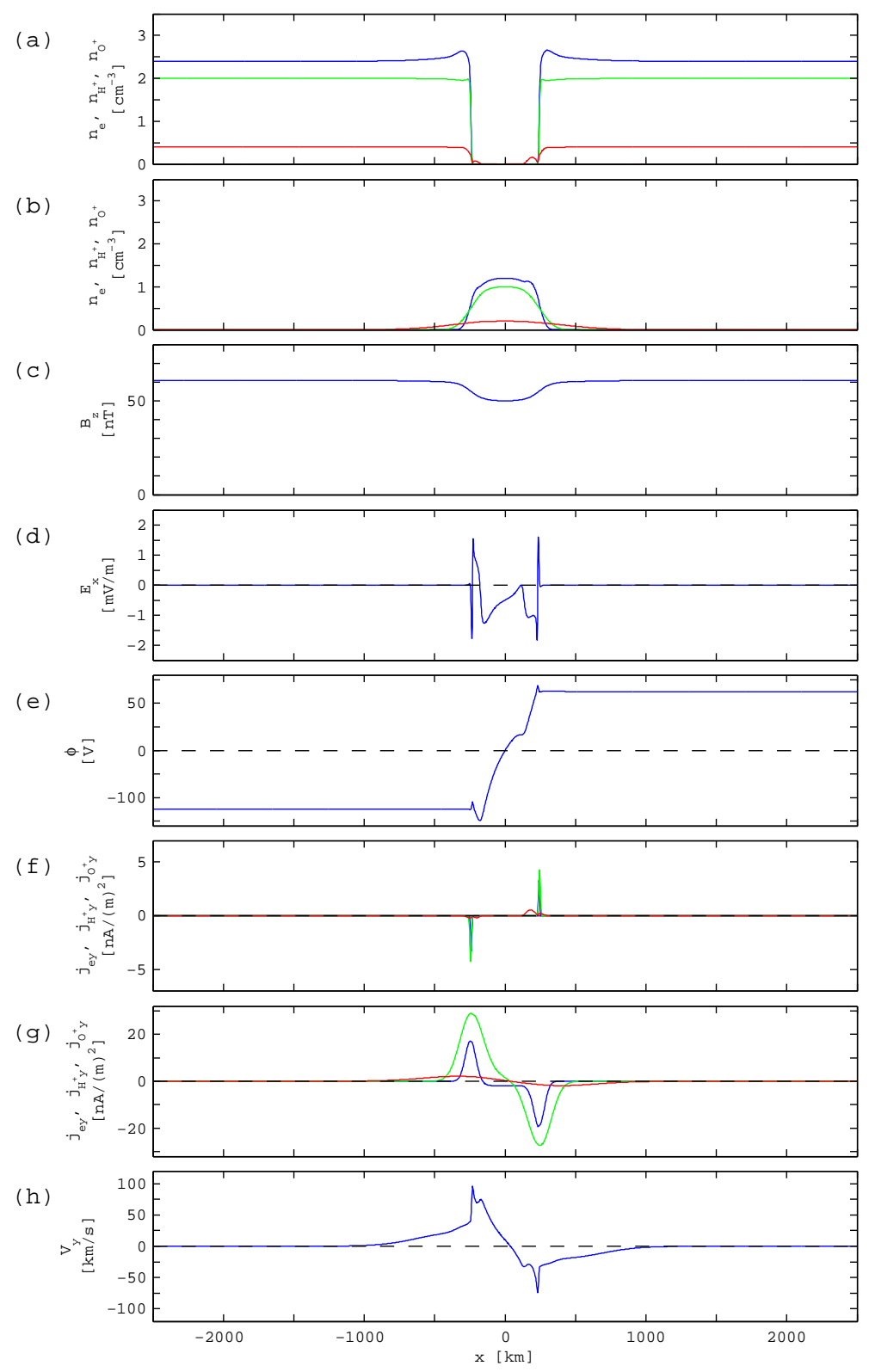

Fig. 9. Plasma and field structure of a $500 \mathrm{~km}$-wide $\mathrm{e}^{-}-\mathrm{H}^{+}-\mathrm{O}^{+}$plasma slab embedded in a uniform background. The plasma parameters are given in Table 1, except for the values of $p_{0}^{(\mathrm{l})}$ and $p_{0}^{(\mathrm{r})}$ that have been reduced by a factor of 4 . There is a cross-field flow $V_{0 y}=10 \mathrm{kms} \mathrm{s}^{-1}$ at the slab centre. The electric potential boundary conditions are $\phi( \pm \infty)= \pm 125 \mathrm{~V}$. The figure format is the same as in Fig. 4 .

and maximum electric field in the slab as a function of $\Delta \phi_{0}$. There are limits to this electric field, about 20 to $30 \mathrm{mV} \mathrm{m}^{-1}$, which is on the order of the slab $\mathrm{H}^{+}$energy expressed in $\mathrm{eV}$ divided by the ion gyroradius: For larger electric fields, thermal ions can no longer be bound by the magnetic field in their gyromotion around the field lines. The electric field limits correspond to limits on the potential difference. An equilibrium is found for $-2050 \mathrm{~V}<\Delta \phi_{0}<+1650 \mathrm{~V}$. The picture is not symmetric, with a larger acceptable range for negative values of $\Delta \phi_{0}$. The maximum acceptable potential differences (1600-2000 V in the present example) are on the order of the hot particle energy $(2000 \mathrm{eV}$ for the hot ions in the slab).

Figure 12 repeats the above computations, but it scans over both $V_{0 y}$ and $\Delta \phi_{0}$. The figure outlines the existence domain: red corresponds to regions where an equilibrium solution exists, while blue indicates that no such equilibrium exists. The domain is symmetric with respect to the sign of $V_{0 y}$. The asymmetry with respect to the sign of $\Delta \phi_{0}$ is evident. There is an interplay between $V_{0 y}$ and $\Delta \phi_{0}$, such that the maximum existence range for $V_{0 y}$ is obtained not for $\Delta \phi_{0}=0$ but for $\Delta \phi_{0} \approx-1250 \mathrm{~V}$. 


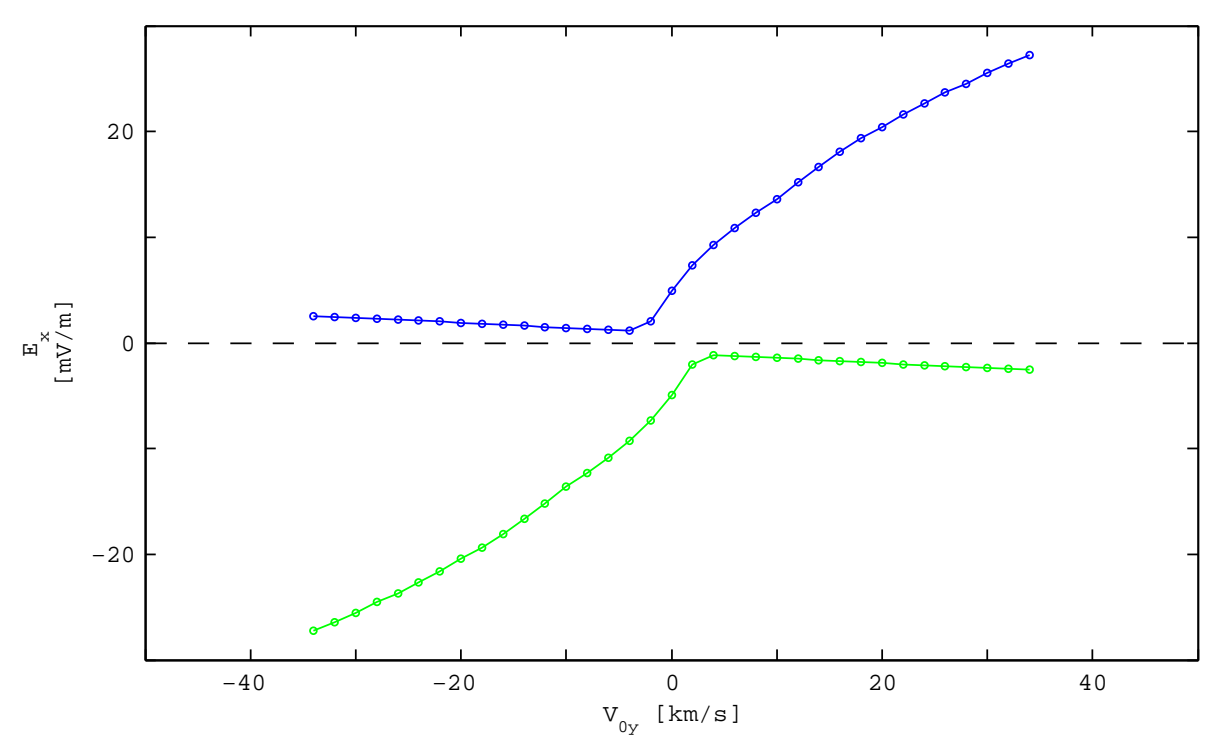

Fig. 10. Minimum (green) and maximum (blue) electric field strength in a $2000 \mathrm{~km}$-wide $\mathrm{e}^{-}-\mathrm{H}^{+}$slab for various values of $V_{0 y}$ in a configuration where $\phi( \pm \infty)=0 \mathrm{~V}$. No equilibrium is found for $\left|V_{0 y}\right|>35 \mathrm{~km} \mathrm{~s}^{-1}$.

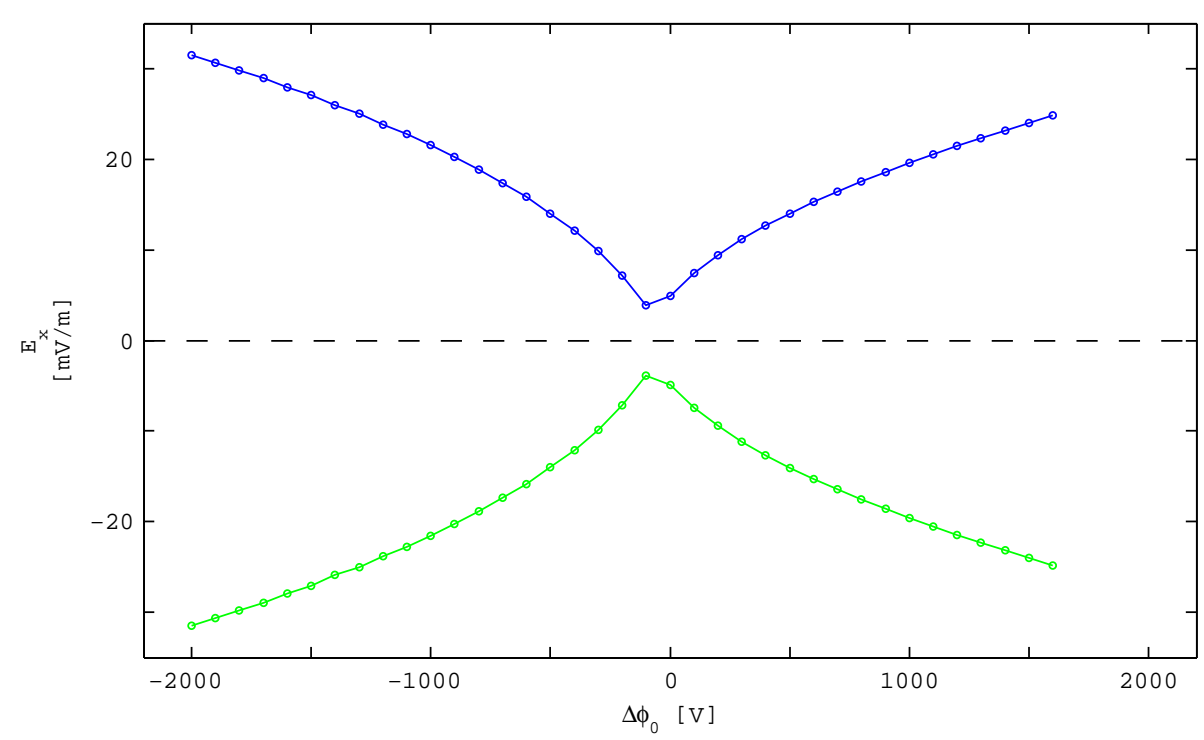

Fig. 11. Minimum (green) and maximum (blue) electric field strength in a $2000 \mathrm{~km}$-wide $\mathrm{e}^{-}-\mathrm{H}^{+}$slab, for various values of $\Delta \phi_{0}=\phi( \pm \infty)$, in a configuration where $V_{0 y}=0 \mathrm{~km} \mathrm{~s}^{-1}$. No equilibrium is found for $\Delta \phi_{0}<-2050 \mathrm{~V}$ or $>+1650 \mathrm{~V}$.

Figure 13 shows the existence domain of the same slab as a function of the flow $\boldsymbol{V}_{0}$ in the slab for a potential difference $\Delta \phi_{0}=0 \mathrm{~V}$ (left) and $1000 \mathrm{~V}$ (right) between slab and background. In contrast with all the calculations presented earlier in this paper, the magnetic field direction may change since $V_{0 z} \neq 0$. This is explained by the fact that the proportion of slab ions to electrons inside the TD interfaces considerably deviates from unity due to the different transition lengths, while the background populations help to ensure quasi-neutrality. The total parallel ion and electron velocities are therefore different in the interfaces, resulting in a net par- allel current. In view of the limited plasma $\beta$, however, the magnetic field rotation is limited, so that the field remains essentially along $z$. The existence domains are symmetric with respect to the sign of the field-aligned flow component $V_{0 z}$, as well as to the sign of the cross-field flow $V_{0 y}$, as can easily be understood from the symmetry of the configuration. The existence domains that are found are all elongated along the magnetic field direction, while their extent in the cross-field direction is limited. This is true for the case $\Delta \phi_{0}=0 \mathrm{kV}$, but even more so for $\Delta \phi_{0}=1 \mathrm{kV}$. The figure also shows, for reference, the slab ion thermal speed. Flow instabilities are 


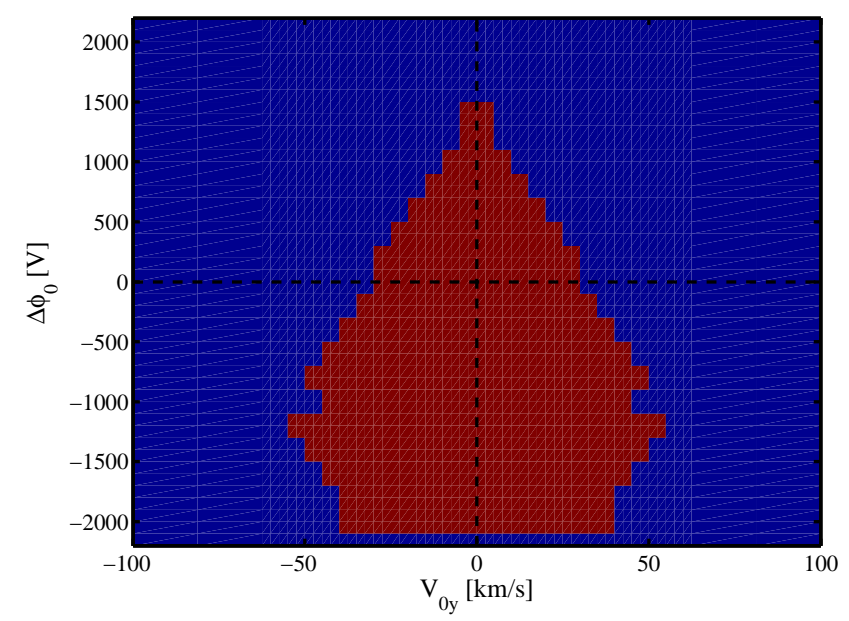

Fig. 12. Two-dimensional scan of the existence domain of a $2000 \mathrm{~km}$-wide $\mathrm{e}^{-}-\mathrm{H}^{+}$slab as a function of the cross-field flow $V_{0 y}$ in the slab and of the potential difference $\Delta \phi_{0}$ between slab and background. Red corresponds to the region where an equilibrium solution is possible.

likely to occur for $V_{0}$ that are larger. Although the equilibrium model described here cannot say anything about such instabilities, it is clear that the model dictates a low transverse flow speed limit above which no equilibrium can be found, at least for the slab configuration presented here with hot slab plasma embedded in a cold background, and with the same electric potential on either side of the slab.

\section{Conclusions}

The present paper discussed a fully self-consistent kinetic tangential discontinuity model of a plasma slab embedded in a uniform background. This is made possible by the use of velocity distributions with two-sided cutoffs (the so-called Type IV distributions); smooth cutoff functions have been used here as a generalisation of earlier work (Echim et al., 2005). Such distributions allow particles to be constrained to a slab of finite width while still retaining the freedom to specify the flow within the slab. An alternative is to constrain particle populations by the electric field they experience in their respective comoving frame as in a Harris-type plasma slab (Harris, 1962), but in that case the slab thickness is directly related to the plasma temperature and the difference between the transverse ion and electron speeds. The computation of the plasma moments of Type I-IV distributions as a function of the electric and magnetic potentials is presented in Appendix A for reference.

The properties of such slabs have been examined here in detail. The essential structural characteristic is the internal electric field of the TD boundaries that is related to thermal effects (different kinetic energies and thus gyroradii of the particles), imposed by external electric potential differences,
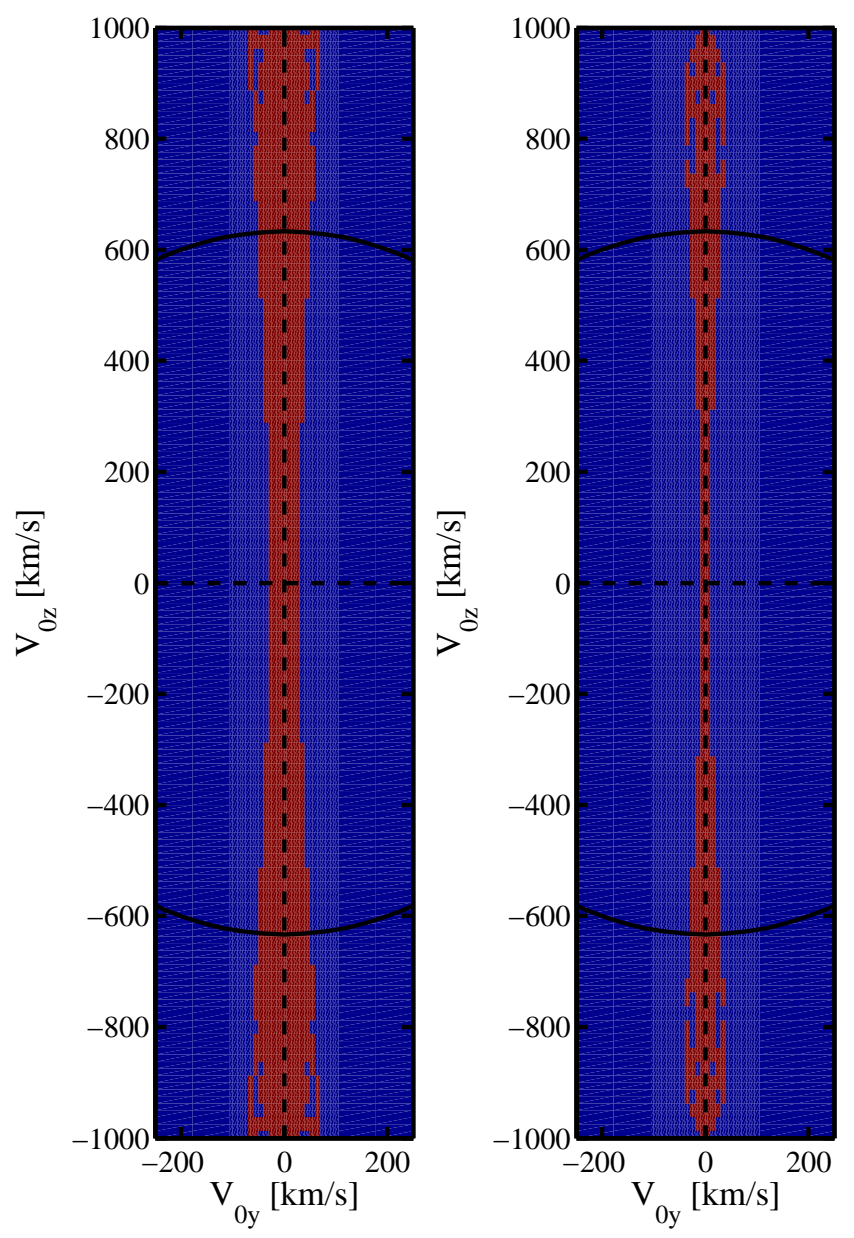

Fig. 13. Two-dimensional scan of the existence domain of a $2000 \mathrm{~km}$-wide $\mathrm{e}^{-}-\mathrm{H}^{+}$slab as a function of the flow $\boldsymbol{V}_{0}$ in the slab for a potential difference $\Delta \phi_{0}=0 \mathrm{~V}$ (left) and $1000 \mathrm{~V}$ (right) between slab and background. Red corresponds to the region where an equilibrium solution is possible. The circle corresponds to the thermal speed of the slab ions $\left(633 \mathrm{~km} \mathrm{~s}^{-1}\right)$. The magnetic field is oriented essentially along $z$.

or produced by plasma convection. In particular the external potential differences and cross-field flow in the slab both may strongly affect the TDs that bound the slab. There are two main conclusions:

- The electric field profile in either interface is different because the convection electric field due to the crossfield flow has the same sign on either side while the charge separation due to different slab and background thermal energies has opposite sign. This leads to an intrinsic asymmetry between the structures of both TDs (Echim et al., 2005). If the slab is connected to the ionosphere, however, additional mechanisms come into play that may result in asymmetries, as the magnetosphereionosphere electric current circuit properties also depend on the precise geometry, on the current-voltage 
relations, and on the ionospheric conductivities (e.g. Lyons, 1980; De Keyser et al., 2010).

- A slab equilibrium exists only for a limited range of cross-field flow speeds and for a limited range of external electric potential differences. This conclusion is strongly related to the effects of external electric potential difference and flow shear across individual planar TD interfaces (De Keyser et al., 1997; De Keyser and Echim, 2013). Such existence limits are absent in models that are restricted to exactly neutral plasma slabs (e.g. Mottez, 2003); in fact, such models do not even allow imposing flow shear or external electric potential differences as boundary conditions.

These conclusions appear to be generic and do not depend too much on the specifics of the model. Even though the model discussed here addresses a simplified configuration, and even if in reality one deals with intrinsically nonequilibrium situations, it is of extreme physical relevance to know for which parameter ranges an equilibrium actually may exist. One should not forget that even if an equilibrium solution appears to exist, it may still be unstable.

The slab problem was discussed here in the context of hot plasma sheet plasma injection into the plasmatrough (e.g. Apatenkov et al., 2007; Zhang et al., 2008). Similar structures may be found elsewhere in planetary magnetospheres, for example

- plasma penetrating into the magnetosphere at the dayside (Marchaudon et al., 2009; Lundin et al., 2003), where the plasma velocity and the associated momentum are of prime importance; in the context of the socalled impulsive penetration mechanism, the electric field inside the slab is called the "polarisation electric field" and it corresponds to charges of opposite sign accumulating in the interfaces on either side (Lemaire, 1977; Lemaire and Roth, 1978; Echim and Lemaire, 2000; Lundin et al., 2003);

- bipolar auroral structures, in particular structures associated with polar cap arcs, often characterised by an electric potential well; in that case the external electric potential differences strongly affect the configuration, while the cross-field flow might be relatively unimportant (Maggiolo et al., 2006, 2011, 2012; De Keyser et al., 2010); and

- plasma fingers, resulting from interchange motion, that penetrate into the plasmasphere (Burch et al., 2005; Mitchell et al., 2009).

The indeterminacy inherent in one-dimensional tangential discontinuities becomes explicit in the models proposed by Sestero (1964, 1966), Roth et al. (1996), and De Keyser and Echim (2013) and in the present paper in the arbitrariness of the choice of distribution functions, and of the cutoff functions in particular. We have already cited observational studies that support at least the qualitative correctness of these models. Nevertheless, one might wish to have more control over the populations trapped inside a TD layer. It is important to realise that the plasma trapped in any TD-type structure can be modelled as a superposition of slabs with plasma distributions of Type IV as introduced here. Including such distributions in a TD model therefore makes it possible to study a much broader set of configurations than before. This can, however, only be done in practice if the accessibility problem is somehow resolved (Whipple et al., 1984) so that one knows what particles are actually "trapped" in the structure.

\section{Appendix A}

\section{Moments of the VDF}

This Appendix gives the analytical expressions for the moments of the velocity distribution introduced by Eq. (4) as a product of a Maxwellian and a particular type of cutoff function. The derivation is analogous to that given by Roth et al. (1996). As we focus on the moments of the VDF of species $s$, we simplify the notation by dropping the subscript $s$. We further assume that the reference frame $x, y, z$ has already been rotated to $x, y^{\prime}, z^{\prime}$ over angle $\theta_{s}$, so that we can also drop the prime. With a VDF written in terms of the invariants of motion as

$f=F\left(H, p_{y}, p_{z}\right) C\left(p_{z}\right)$,

and with

$$
\begin{aligned}
F & =N\left(\frac{m}{2 \pi \kappa}\right)^{\frac{3}{2}} \mathrm{e}^{-\left(H+\frac{m \mathcal{V}^{2}}{2}-p_{y} \mathcal{V}_{y}-p_{z} \mathcal{V}_{z}\right) / \kappa}, \\
H_{0} & =\frac{m}{2}\left(v_{y}^{2}+v_{z}^{2}\right)+Z e \phi \\
v_{x} & = \pm \sqrt{\frac{2}{m}\left(H-H_{0}\right)} \\
v_{y} & =\left(p_{y}-Z e A_{y}\right) / m, \\
v_{z} & =\left(p_{z}-Z e A_{z}\right) / m,
\end{aligned}
$$

it is possible to express the moments as integrals over the invariants of motion. As $v_{x}$ can be both positive and negative, one finds

$$
\begin{aligned}
Q^{(i j k)} & =\int_{-\infty}^{+\infty} \int_{-\infty}^{+\infty} \int_{-\infty}^{+\infty} v_{x}^{i} v_{y}^{j} v_{z}^{k} f \mathrm{~d} v_{x} \mathrm{~d} v_{y} \mathrm{~d} v_{z} \\
& =2 \int_{-\infty}^{+\infty} \int_{-\infty}^{+\infty} \int_{H_{0}}^{+\infty} \frac{v_{x}^{i} v_{y}^{j} v_{z}^{k} f}{m^{2} \sqrt{2 m\left(H-H_{0}\right)}} \mathrm{d} H \mathrm{~d} p_{y} \mathrm{~d} p_{z}
\end{aligned}
$$

for $i$ even; $Q^{(i j k)}=0$ for $i$ odd. Here, $v_{x}\left(H, p_{y}, p_{z}\right), v_{y}\left(p_{y}\right)$, $v_{z}\left(p_{z}\right), f\left(H, p_{y}, p_{z}\right)$, and $H_{0}\left(p_{y}, p_{z}\right)$ are all functions of the 
invariants. First perform the integration over $H$ as

$$
\begin{aligned}
Q^{(i j k)}= & 2^{\frac{i-2}{2}} \mathcal{N}(\pi \kappa)^{-\frac{3}{2}} m^{-\frac{i+2 j+2 k+2}{2}} \\
& \int_{-\infty}^{+\infty} \int_{-\infty}^{+\infty}\left(p_{y}-Z e A_{y}\right)^{j}\left(p_{z}-Z e A_{z}\right)^{k} C\left(p_{z}\right) \\
\mathcal{I}_{H}^{(i)}= & \int_{H_{0}}^{+\left(\frac{m \mathcal{V}^{2}}{2}-p_{y} \mathcal{V}_{y}-p_{z} \mathcal{V}_{z}\right) / \kappa} \mathrm{e}_{H}^{-H / \kappa}\left(p_{y}, p_{z}\right) \mathrm{d} p_{y} \mathrm{~d} p_{z}, \\
= & \frac{i !}{(i / 2) ! 2^{i}} \kappa^{\frac{i+1}{2}} \mathrm{e}^{-H_{0}\left(p_{y}, p_{z}\right) / \kappa} .
\end{aligned}
$$

The moment can then be written as a product of two integrals over $p_{y}$ and over $p_{z}$ :

$$
\begin{aligned}
Q^{(i j k)}= & \mathcal{M}^{(i j k)} \mathcal{I}_{p_{y}}^{(j)} \mathcal{I}_{p_{z}}^{(k)}, \\
\mathcal{M}^{(i j k)}= & \mathcal{N} \frac{i !}{2^{\frac{i+2}{2}} \pi(i / 2) !} \frac{\kappa^{\frac{i-2}{2}}}{\frac{i+2 j+2 k+2}{2}} \mathrm{e}^{-\left(m \mathcal{V}^{2} / 2+Z e \phi\right) / \kappa}, \\
\mathcal{I}_{p_{y}}^{(j)}= & \int_{-\infty}^{+\infty}\left(p_{y}-Z e A_{y}\right)^{j} \mathrm{e}^{\left[p_{y} \mathcal{V}_{y}-\frac{\left(p_{y}-Z e A_{y}\right)^{2}}{2 m}\right] / \kappa} \mathrm{d} p_{y}, \\
\mathcal{I}_{p_{z}}^{(k)}= & \int_{-\infty}^{+\infty}\left(p_{z}-Z e A_{z}\right)^{k} C\left(p_{z}\right) \mathrm{e}^{\left[p_{z} \mathcal{V}_{z}-\frac{\left(p_{z}-Z e A_{z}\right)^{2}}{2 m}\right] / \kappa} \mathrm{d} p_{z} .
\end{aligned}
$$

Introduce

$P_{y}=\left[p_{y}-\left(m \mathcal{V}_{y}+Z e A_{y}\right)\right] / \gamma$

$P_{z}=\left[p_{z}-\left(m \mathcal{V}_{z}+Z e A_{z}\right)\right] / \gamma$,

and

$P_{0}^{(1)}=\left[s p_{0}^{(1)}-\left(m \mathcal{V}_{z}+Z e A_{z}\right)\right] / \gamma$,

$P_{0}^{(r)}=\left[s p_{0}^{(r)}-\left(m \mathcal{V}_{z}+Z e A_{z}\right)\right] / \gamma$,

with $\gamma=\sqrt{2 m \kappa}$. Using the dimensionless momentum $\mathcal{W}_{y, z}=m \mathcal{V}_{y, z} / \gamma$, the integrals can be transformed to

$$
\begin{aligned}
\mathcal{I}_{p_{y}}^{(j)}= & \gamma^{j+1} \mathrm{e}^{\left(m \mathcal{V}_{y}^{2} / 2+Z e A_{y} \mathcal{V}_{y}\right) / \kappa} \\
& \cdot I^{(j)}\left(\mathcal{W}_{y}\right) \\
\mathcal{I}_{p_{z}}^{(k)}= & \gamma^{k+1} \mathrm{e}^{\left(m \mathcal{V}_{z}^{2} / 2+Z e A_{z} \mathcal{V}_{z}\right) / \kappa} \\
& \cdot\left[v^{(1)} I_{-}^{(k)}\left(\mathcal{W}_{z}\right)+v^{(r)} I_{+}^{(k)}\left(\mathcal{W}_{z}\right)-v^{(c)} I^{(k)}\left(\mathcal{W}_{z}\right)\right],
\end{aligned}
$$

where

$$
\begin{aligned}
I^{(j)}(\mathcal{W}) & =\int_{-\infty}^{+\infty}(P+\mathcal{W})^{j} \mathrm{e}^{-P^{2}} \mathrm{~d} P \\
I_{+}^{(k)}(\mathcal{W}) & =\int_{-\infty}^{+\infty}(P+\mathcal{W})^{k} \frac{\mathrm{e}^{-P^{2}}}{2} \operatorname{erfc}\left(+\frac{P-P_{0}^{(r)}-\mathcal{W}}{s \sqrt{\ell^{(r)^{2}}-1}}\right) \mathrm{d} P \\
I_{-}^{(k)}(\mathcal{W}) & =\int_{-\infty}^{+\infty}(P+\mathcal{W})^{k} \frac{\mathrm{e}^{-P^{2}}}{2} \operatorname{erfc}\left(-\frac{P-P_{0}^{(1)}-\mathcal{W}}{s \sqrt{\ell^{(1)^{2}}-1}}\right) \mathrm{d} P .
\end{aligned}
$$

Using the binomial theorem, $I^{(j)}(\mathcal{W})$ is found as

$$
I^{(j)}=\sum_{\xi=0}^{j} \frac{j !}{\xi !(j-\xi) !} \mathcal{W}^{j-\xi} E^{(\xi)},
$$

where

$$
E^{(\xi)}=\int_{-\infty}^{+\infty} t^{\xi} \mathrm{e}^{-t^{2}} \mathrm{~d} t
$$

Since $E^{(0)}=\sqrt{\pi}, E^{(1)}=0$, and $E^{(2)}=\sqrt{\pi} / 2$, one finds

$$
\begin{aligned}
& I^{(0)}=\sqrt{\pi}, \\
& I^{(1)}=\sqrt{\pi} \mathcal{W}, \\
& I^{(2)}=\frac{\sqrt{\pi}}{2}\left(1+2 \mathcal{W}^{2}\right) .
\end{aligned}
$$

Using the binomial theorem for $I_{ \pm}^{(k)}(\mathcal{W})$ gives

$$
I_{ \pm}^{(k)}=\sum_{\xi=0}^{k} \frac{k !}{\xi !(k-\xi) !} \mathcal{W}^{k-\xi} E_{ \pm}^{(\xi)}
$$

where

$$
\begin{aligned}
& E_{ \pm}^{(\xi)}=\frac{1}{2} \int_{-\infty}^{+\infty} t^{\xi} \mathrm{e}^{-t^{2}} \operatorname{erfc}\left(\alpha_{ \pm} t+\beta_{ \pm}\right) \mathrm{d} t \\
& \alpha_{ \pm}= \pm \frac{1}{s \sqrt{\ell^{2}-1}}, \quad \beta_{ \pm}=\mp \frac{P_{0}+\mathcal{W}}{s \sqrt{\ell^{2}-1}}
\end{aligned}
$$

This results in

$$
\begin{aligned}
& E_{ \pm}^{(0)}=\frac{\sqrt{\pi}}{2} \operatorname{erfc}\left(\mathcal{R}_{ \pm}\right) \\
& E_{ \pm}^{(1)}=-\frac{\mathcal{S}_{ \pm}}{2} \exp \left(-\mathcal{R}_{ \pm}^{2}\right) \\
& E_{ \pm}^{(2)}=\frac{\sqrt{\pi}}{4} \operatorname{erfc}\left(\mathcal{R}_{ \pm}\right)+\frac{\mathcal{R}_{ \pm}}{2} \mathcal{S}_{ \pm}^{2} \exp \left(-\mathcal{R}_{ \pm}^{2}\right)
\end{aligned}
$$


where

$$
\begin{aligned}
\mathcal{R}_{+} & =\beta_{+} / \sqrt{1+\alpha_{+}^{2}} \\
& =-\left(P_{0}^{(r)}+\mathcal{W}\right) / s \ell^{(r)}=-\left(p_{0}^{(r)}-|Z| e A_{z}\right) / \gamma \ell^{(r)}, \\
\mathcal{S}_{+} & =\alpha_{+} / \sqrt{1+\alpha_{+}^{2}}=1 / s \ell^{(r)}, \\
\mathcal{R}_{-} & =-\beta_{-} / \sqrt{1+\alpha_{-}^{2}} \\
& =\left(P_{0}^{(1)}+\mathcal{W}\right) / s \ell^{(1)}=\left(p_{0}^{(1)}-|Z| e A_{z}\right) / \gamma \ell^{(1)}, \\
\mathcal{S}_{-} & =-\alpha_{-} / \sqrt{1+\alpha_{-}^{2}}=-1 / s \ell^{(1)} .
\end{aligned}
$$

One thus obtains

$$
\begin{aligned}
& I_{ \pm}^{(0)}=\frac{\sqrt{\pi}}{2} \operatorname{erfc}\left(\mathcal{R}_{ \pm}\right) \\
& I_{ \pm}^{(1)}=\frac{\sqrt{\pi}}{2} \operatorname{erfc}\left(\mathcal{R}_{ \pm}\right) \mathcal{W}-\frac{\mathcal{S}_{ \pm}}{2} \mathrm{e}^{-\mathcal{R}_{ \pm}^{2}} \\
& I_{ \pm}^{(2)}=\frac{\sqrt{\pi}}{4} \operatorname{erfc}\left(\mathcal{R}_{ \pm}\right)\left(1+2 \mathcal{W}^{2}\right)+\frac{\mathcal{S}_{ \pm}}{2}\left(\mathcal{R}_{ \pm} \mathcal{S}_{ \pm}-2 \mathcal{W}\right) \mathrm{e}^{-\mathcal{R}_{ \pm}^{2}} .
\end{aligned}
$$

Note that integrals $E^{(\xi)}$ and $E_{ \pm}^{(\xi)}$, and $I^{(\xi)}$ and $I_{ \pm}^{(\xi)}$, are related by

$$
\begin{aligned}
& \lim _{\ell \rightarrow+\infty} 2 E_{ \pm}^{(\xi)}=E^{(\xi)} \\
& \lim _{\ell \rightarrow+\infty} 2 I_{ \pm}^{(\xi)}=I^{(\xi)}
\end{aligned}
$$

Any moment can now be computed; most plasma parameters of interest are obtained from moments of order $i+j+k \leq 2$. Expressing the moments in terms of their original parameters, we find

$$
\begin{aligned}
Q^{(000)}= & \mathcal{E}\left(v^{(1)} \mathcal{G}^{(1)}+v^{(r)} \mathcal{G}^{(r)}-v^{(c)}\right), \\
Q^{(010)}= & \mathcal{V}_{y} Q^{(000)}, \\
Q^{(001)}= & \mathcal{V}_{z} Q^{(000)}+s \sqrt{\frac{2 \kappa}{m}}\left(v^{(1)} \mathcal{H}^{(1)}-v^{(r)} \mathcal{H}^{(r)}\right) \mathcal{E}, \\
Q^{(200)}= & \frac{\kappa}{m} Q^{(000)} \\
Q^{(020)}= & \left(\frac{\kappa}{m}+\mathcal{V}_{y}^{2}\right) Q^{(000)} \\
Q^{(002)}= & \left(\frac{\kappa}{m}+\mathcal{V}_{z}^{2}\right) Q^{(000)} \\
& +\frac{2 \kappa}{m} \mathcal{E}\left(v^{(1)} \mathcal{H}^{(1)} \mathcal{K}^{(1)}-v^{(r)} \mathcal{H}^{(r)} \mathcal{K}^{(r)}\right),
\end{aligned}
$$

where

$$
\begin{aligned}
\mathcal{E} & =\mathcal{N} \exp \left(-\frac{Z e}{\kappa}\left(\phi-A_{y} \mathcal{V}_{y}-A_{z} \mathcal{V}_{z}\right)\right), \\
\mathcal{G}^{(1)} & =\frac{1}{2} \operatorname{erfc}\left(\frac{p_{0}^{(1)}-|Z| e A_{z}}{\gamma \ell^{(1)}}\right), \\
\mathcal{G}^{(r)} & =\frac{1}{2} \operatorname{erfc}\left(-\frac{p_{0}^{(r)}-|Z| e A_{z}}{\gamma \ell^{(r)}}\right), \\
\mathcal{H}^{(1)} & =\mathrm{e}^{-\left(p_{0}^{(1)}-|Z| e A_{z}\right)^{2} / \gamma^{2} \ell^{(1)}{ }^{2}} / 2 \sqrt{\pi} \ell^{(1)}, \\
\mathcal{H}^{(r)} & =\mathrm{e}^{-\left(p_{0}^{(r)}-|Z| e A_{z}\right)^{2} / \gamma^{2} \ell^{(r)^{2}} / 2 \sqrt{\pi} \ell^{(r)},} \\
\mathcal{K}^{(1)} & =\left(p_{0}^{(1)}-|Z| e A_{z}+2 s \ell^{(1)}{ }^{2} m \mathcal{V}_{z}\right) / \gamma \ell^{(1)^{2}}, \\
\mathcal{K}^{(r)} & =\left(p_{0}^{(r)}-|Z| e A_{z}+2 s \ell^{(r)^{2}} m \mathcal{V}_{z}\right) / \gamma \ell^{(r)^{2}} .
\end{aligned}
$$

The plasma parameters can now be calculated explicitly:

$$
\begin{aligned}
& n=Q^{(000)} \text {, } \\
& V_{y}=Q^{(010)} / Q^{(000)}=\mathcal{V}_{y} \text {, } \\
& j_{y}=Z e Q^{(010)}=Z e n \mathcal{V}_{y} \text {, } \\
& V_{z}=Q^{(001)} / Q^{(000)} \\
& =\mathcal{V}_{z}+s \sqrt{\frac{2 \kappa}{m}}\left(v^{(1)} \mathcal{H}^{(1)}-v^{(r)} \mathcal{H}^{(r)}\right) \mathcal{E} / n, \\
& j_{z}=Z e Q^{(001)} \\
& =Z e n \mathcal{V}_{z}+|Z| e \sqrt{\frac{2 \kappa}{m}}\left(v^{(1)} \mathcal{H}^{(1)}-v^{(r)} \mathcal{H}^{(r)}\right) \mathcal{E}, \\
& T_{x}=\frac{m}{k_{B}} \frac{Q^{(200)}}{Q^{(000)}}=\mathcal{T} \text {, } \\
& T_{y}=\frac{m}{k_{B}}\left[\frac{Q^{(020)}}{Q^{(000)}}-\left(\frac{Q^{(010)}}{Q^{(000)}}\right)^{2}\right]=\mathcal{T}, \\
& T_{z}=\frac{m}{k_{B}}\left[\frac{Q^{(002)}}{Q^{(000)}}-\left(\frac{Q^{(001)}}{Q^{(000)}}\right)^{2}\right] \\
& =\mathcal{T}\left(1+\frac{m}{\kappa}\left(\mathcal{V}_{z}^{2}-V_{z}^{2}\right)\right. \\
& \left.+\frac{2 \mathcal{E}}{n}\left(v^{(1)} \mathcal{H}^{(1)} \mathcal{K}^{(1)}-v^{(r)} \mathcal{H}^{(r)} \mathcal{K}^{(r)}\right)\right) .
\end{aligned}
$$

It can be shown that these formulae reduce to the corresponding ones in Roth et al. (1996) for distributions of types I, II, and III.

Taking the total derivative of the quasi-neutrality condition relative to $x$,

$\frac{\mathrm{d}}{\mathrm{d} x} \sum_{s} Z_{s} e n^{(s)}\left(\phi(x), A_{y}(x), A_{z}(x)\right)=0$,

produces an expression for the electric field,

$$
E_{x}=\left[B_{z} \sum_{s} Z_{s} \frac{\partial n^{(s)}}{\partial A_{y}}-B_{y} \sum_{s} Z_{s} \frac{\partial n^{(s)}}{\partial A_{z}}\right] / \sum_{s} Z_{s} \frac{\partial n^{(s)}}{\partial \phi},
$$


where (dropping the index)

$$
\begin{aligned}
\frac{\partial n}{\partial \phi} & =-\frac{Z e}{\kappa} n, \\
\frac{\partial n}{\partial A_{y}} & =\frac{Z e}{\kappa} n \mathcal{V}_{y}, \\
\frac{\partial n}{\partial A_{z}} & =\frac{Z e}{\kappa} n \mathcal{V}_{z}+|Z| e \sqrt{\frac{2}{m \kappa}} \mathcal{E}\left(v^{(1)} \mathcal{H}^{(1)}-v^{(r)} \mathcal{H}^{(r)}\right) .
\end{aligned}
$$

This expression allows us to obtain the electric field without using the Poisson equation explicitly.

Acknowledgements. This work was supported by the Belgian Science Policy Office through Prodex/CLUSTER (PEA4200090316).

Topical Editor L. Blomberg thanks A. Artemyev and one anonymous referee for their help in evaluating this paper.

\section{References}

Angelopoulos, V., Baumjohann, W., Kennel, C. F., Coroniti, F. V., Kivelson, M. G., Pellat, R., Walker, R. J., Lühr, H., and Paschmann, G.: Bursty bulk flows in the inner central plasma sheet, J. Geophys. Res., 97, 4027-4039, 1992.

Apatenkov, S. V., Sergeev, V. A., Kubyshkina, M. V., Nakamura, R., Baumjohann, W., Runov, A., Alexeev, I., Fazakerley, A., Frey, H., Muhlbachler, S., Daly, P. W., Sauvaud, J.-A., Ganushkina, N., Pulkkinen, T., Reeves, G. D., and Khotyaintsev, Y.: Multi-spacecraft observation of plasma dipolarization/injection in the inner magnetosphere, Ann. Geophys., 25, 801-814, doi:10.5194/angeo-25-801-2007, 2007..

Artemyev, A. V.: A model of one-dimensional current sheet with parallel currents and normal component of magnetic field, Phys. Plasmas, 18, 022104, doi:10.1063/1.3552141, 2011.

Burch, J. L., Goldstein, J., Hill, T. W., Young, D. T., Crary, F. J., Coates, A. J., André, N., Kurth, W. S., and Sittler Jr., E. C.: Properties of local plasma injections in Saturn's magnetosphere, Geophys. Res. Lett., 32, L14S02, doi:10.1029/2005GL022611, 2005.

De Keyser, J. and Echim, M.: Electric potential differences across auroral generator interfaces, Ann. Geophys., 31, 251-261, doi:10.5194/angeo-31-251-2013, 2013.

De Keyser, J., Roth, M., Lemaire, J., Tsurutani, B., Ho, C., and Hammond, C.: Theoretical plasma distributions consistent with Ulysses magnetic field observations in a high-speed solar wind tangential discontinuity, Solar Phys., 166, 415-422, 1996.

De Keyser, J., Roth, M., Tsurutani, B., Ho, C., and Phillips, J.: Solar wind velocity jumps across tangential discontinuities: Ulysses observations and kinetic interpretation, A\&A, 321, 945-959, 1997.

De Keyser, J., Maggiolo, R., and Echim, M.: Monopolar and bipolar auroral electric fields and their effects, Ann. Geophys., 28, 20272046, doi:10.5194/angeo-28-2027-2010, 2010.

Deng, X. H., Tang, R. X., Nakamura, R., Baumjohann, W., Zhang, T. L., Daly, P. W., Rème, H., Carr, C. M., Balogh, A., Liu, Z. X., and Wang, J. F.: Observation of reconnection pulses by Cluster and Double Star, Ann. Geophys., 23, 2921-2927, doi:10.5194/angeo-23-2921-2005, 2005.
Echim, M. and Lemaire, J. F.: Laboratory and numerical simulations of the impulsive penetration mechanism, Space Sci. Rev., 92, 565-601, 2000.

Echim, M., Lemaire, J. F., and Roth, M.: Self-consistent solution for a collisionless plasma slab in motion across a magnetic field, Phys. Plasmas, 12, 072904, doi:10.1063/1.1943848, 2005.

Echim, M. M., Maggiolo, R., Roth, M., and De Keyser, J.: A magnetospheric generator driving ion and electron acceleration and electric currents in a discrete auroral arc observed by Cluster and DMSP, Geophys. Res. Lett., 36, L12111, doi:10.1029/2009GL038343, 2009.

Echim, M., Maggiolo, R., De Keyser, J., Zhang, T. L., Voitcu, G., Barabash, S., and Lundin, R.: Comparative investigation of the Terrestrial and Venusian magnetopause: kinetic modeling and experimental observations by Cluster and Venus Express, Planet. Space Sci., 59, 1028-1038, doi:10.1016/j.pss.2010.04.019, 2011

Harris, E. G.: On a plasma sheath separating regions of oppositely directed magnetic field, Il Nuovo Cimento, 23, 115-121, 1962.

Hubert, D., Harvey, C., Roth, M., and De Keyser, J.: Electron density at the subsolar magnetopause for high magnetic shear: ISEE 1 and 2 observations, J. Geophys. Res., 103, 6685-6692, 1998.

Lee, L. C. and Kan, J. R.: A unified kinetic model of the tangential magnetopause structure, J. Geophys. Res., 84, 6417-6426, doi:10.1029/JA084iA11p06417, 1979.

Lemaire, J.: Impulsive penetration of filamentary plasma elements into the magnetospheres of the Earth and Jupiter, Planet Space Sci., 25, 887-890, 1977.

Lemaire, J. and Roth, M.: Penetration of solar wind plasma elements into the magnetosphere, J. Atmos. Terr. Phys., 40, 331335, 1978.

Lundin, R., Sauvaud, J.-A., Rème, H., Balogh, A., Dandouras, I., Bosqued, J. M., Carlson, C., Parks, G. K., Möbius, E., Kistler, L. M., Klecker, B., Amata, E., Formisano, V., Dunlop, M., Eliasson, L., Korth, A., Lavraud, B., and McCarthy, M.: Evidence for impulsive solar wind plasma penetration through the dayside magnetopause, Ann. Geophys., 21, 457-472, doi:10.5194/angeo-21457-2003, 200.

Lyons, L. R.: Generation of large-scale regions of auroral currents, electric potentials and precipitation by the divergence of the convection electric field, J. Geophys. Res., 85, 17-24, 1980.

Maggiolo, R., Sauvaud, J. A., Fontaine, D., Teste, A., Grigorenko, E., Balogh, A., Fazakerley, A., Paschmann, G., Delcourt, D., and Rème, H.: A multi-satellite study of accelerated ionospheric ion beams above the polar cap, Ann. Geophys., 24, 1665-1684, doi:10.5194/angeo-24-1665-2006, 2006.

Maggiolo, R., Echim, M., De Keyser, J., Fontaine, D., Jacquey, C., and Dandouras, I.: Polar cap ion beams during periods of northward IMF: Cluster statistical results, Ann. Geophys., 29, 771787, doi:10.5194/angeo-29-771-2011, 2011.

Maggiolo, R., Echim, M., Simon Wedlund, C., Zhang, Y., Fontaine, D., Lointier, G., and Trotignon, J.-G.: Polar cap arcs from the magnetosphere to the ionosphere: kinetic modelling and observations by Cluster and TIMED, Ann. Geophys., 30, 283-302, doi:10.5194/angeo-30-283-2012, 2012.

Marchaudon, A., Cerisier, J.-C., Dunlop, M. W., Pitout, F., Bosqued, J.-M., and Fazakerley, A. N.: Shape, size, velocity and fieldaligned currents of dayside plasma injections: a multi-altitude study, Ann. Geophys., 27, 1251-1266, doi:10.5194/angeo-271251-2009, 2009. 
Mitchell, D. G., Carbary, J. F., Cowley, S. W. H., Hill, T. W., and Zarka, P.: The Dynamics of Saturn's Magnetosphere, in: Saturn from Cassini-Huygens, edited by: Dougherty, M. K., pp. 257279, Springer, doi:10.1007/978-1-4020-9217-6_10, 2009.

Mottez, F.: Exact nonlinear analytic Vlasov-Maxwell tangential equilibria with arbitrary density and temperature profiles, Phys. Plasmas, 10, 2501-2508, 2003.

Nicholson, R. B.: Solution of the Vlasov Equations for a Plasma in an Externally Uniform Magnetic Field, Phys. Fluids, 6, 15811586, 1963.

Reasoner, D. L., Craven, P. D., and Chappell, C. R.: Characteristics of low-energy plasma in the plasmasphere and plasma trough, J. Geophys. Res., 88, 7913-7925, doi:10.1029/JA088iA10p07913, 1983.

Roth, M., De Keyser, J., and Kuznetsova, M. M.: Vlasov theory of the equilibrium structure of tangential discontinuities in space plasmas, Space Sci. Rev., 76, 251-317, 1996.
Sestero, A.: Structure of plasma sheaths, Phys. Fluids, 7, 44-51, 1964.

Sestero, A.: Vlasov equation study of plasma motion across magnetic fields, Phys. Fluids, 9, 2006-2013, 1966.

Sojka, J. J., Schunk, R. W., Johnson, J. F. E., Waite, J. H., and Chappell, C. R.: Characteristics of thermal and suprathermal ions associated with the dayside plasma trough as measured by the Dynamics Explorer Retarding Ion Mass Spectrometer, J. Geophys. Res., 88, 7895-7911, doi:10.1029/JA088iA10p07895, 1983.

Whipple, E. C., Hill, J. R., and Nichols, J. D.: Magnetopause structure and the question of particle accessibility, J. Geophys. Res., 89, 1508-1516, doi:10.1029/JA089iA03p01508, 1984.

Zhang, J.-C., Wolf, R. A., Sazykin, S., and Toffoletto, F. R.: Injection of a bubble into the inner magnetosphere, Geophys. Res. Lett., 35, L02110, doi:10.1029/2007GL032048, 2008. 